Neil MacBeth

Anna Trullenque-Eriksson

Nikolaos Donos

Nikos Mardas

Review

\title{
Hard and soft tissue changes following alveolar ridge preservation: a systematic review
}

Key words: alveolar ridge preservation, gingival tissue, histology, tooth extraction

Authors' affiliations:

Neil MacBeth, Defence Primary Health Care and

Centre for Oral Clinical Research, Institute of

Dentistry, Barts \& The London School of Medicine

\& Dentistry, Queen Mary University of London

(QMUL), London, UK

Anna Trullenque-Eriksson, Nikolaos Donos, Centre for Oral Clinical Research, Institute of Dentistry,

Barts \& The London School of Medicine \&

Dentistry, Queen Mary University of London

(QMUL), London, UK

Nikos Mardas, Center for Adult Health Sciences,

Institute of Dentistry, Barts \& The London School

of Medicine \& Dentistry, Queen Mary University

of London (QMUL), London, UK

Corresponding author:

Professor Nikos Donos, DDS, MS, FHEA,

FDSRCSEngl., PhD

Head of Centre for Oral Clinical Research, Institute of Dentistry, Barts \& The London School of

Medicine \& Dentistry, Queen Mary University of

London (QMUL), Turner Street, London E1 2AD, UK

Tel.: +44 (0) 2078823063

e-mail: n.donos@qmul.ac.uk

\section{Abstract}

Objective: Two focused questions were addressed within this systematic review. Q1) What is the effect of alveolar ridge preservation on linear and volumetric alveolar site dimensions, keratinised measurements, histological characteristics and patient-based outcomes when compared to unassisted socket healing. Q2) What is the size effect of these outcomes in three different types of intervention (guided bone regeneration, socket grafting and socket seal).

Materials and methods: An electronic search (MEDLINE, EMBASE, Cochrane Central Register LILACS, Web of Science) and hand-search was conducted up to June 2015. Randomised controlled trials (RCT) and controlled clinical trials (CCT); with unassisted socket healing as controls: were eligible in the analysis for Q1. RCTs, CCTs and large prospective case series with or without an unassisted socket healing as control group were eligible in the analysis for Q2.

Results: Nine papers (8 RCTs and 1 CCTs) were included in the analysis for Q1 and 37 papers (29 RCTs, 7 CCTs and 1 case series) for Q2. The risk for bias was unclear or high in most of the studies. Q1: the standardised mean difference (SMD) in vertical mid-buccal bone height between ARP and a non-treated site was $0.739 \mathrm{~mm}(95 \% \mathrm{Cl}: 0.332$ to 1.147$)$. The SMD when proximal vertical bone height and horizontal bone width was compared was $0.796 \mathrm{~mm}$ ( $95 \% \mathrm{Cl}:-1.228$ to 0.364$)$ and $1.198 \mathrm{~mm}$ ( $95 \% \mathrm{Cl}:-0.0374$ to 2.433 ). Examination of ARP sites revealed significant variation in vital and trabecular bone percentages and keratinised tissue width and thickness. Adverse events were routinely reported, with three papers reporting a high level of complications in the test and control groups and two papers reporting greater risks associated with ARP. No studies reported on variables associated with the patient experience in either the test or the control group. Q2: A pooled effect reduction (PER) in mid-buccal alveolar ridge height of $-0.467 \mathrm{~mm}(95 \% \mathrm{Cl}:-0.866$ to $-0.069)$ was recorded for $\mathrm{GBR}$ procedures and $-0.157 \mathrm{~mm}(95 \% \mathrm{Cl}:-0.554$ to 0.239$)$ for socket grafting. A proximal vertical bone height reduction of $-0.356 \mathrm{~mm}(95 \% \mathrm{Cl}:-0.490$ to -0.222$)$ was recorded for GBR, with a horizontal dimensional reduction of $-1.45 \mathrm{~mm}(95 \% \mathrm{Cl}:-1.892$ to $-1.008)$ measured following GBR and $-1.613 \mathrm{~mm}(95 \% \mathrm{Cl}:-1.989$ to -1.238$)$ for socket grafting procedures. Five papers reported on histological findings after ARP. Two papers indicated an increase in the width of the keratinised tissue following GBR, with two papers reporting a reduction in the thickness of the keratinised tissue following GBR. Histological examination revealed extensive variations in the treatment protocols and biomaterials materials used to evaluate extraction socket healing. GBR studies reported a variation in total bone formation of $47.9 \pm 9.1 \%$ to $24.67 \pm 15.92 \%$. Post-operative complications were reported by 29 papers, with the most common findings soft tissue inflammation and infection.

Conclusion: ARP results in a significant reduction in the vertical bone dimensional change following tooth extraction when compared to unassisted socket healing. The reduction in horizontal alveolar bone dimensional change was found to be variable. No evidence was identified to clearly indicate the superior impact of a type of ARP intervention (GBR, socket filler and socket seal) on bone dimensional preservation, bone formation, keratinised tissue dimensions and patient complications.

Alveolar bone and soft tissue remodelling is a normal physiological response following tooth extraction. These tissue changes have been recorded as leading to a $40-60 \%$ decrease in the height and the width of the residual alveolar ridge (Johnson 1969; Farmer \& Darby 2014), narrowing of the keratinised mucosa and reduction in the volumetric soft

preservation: a systematic review.

Clin. Oral Impl. Res. 00, 2016, 1-22

doi: $10.1111 /$ clr. 12911 
tissue thickness (Tarnow et al. 1996; Schropp et al. 2005; Darby et al. 2009; Thoma et al. 2009). The resorption process varies greatly amongst individual patients and tooth position and may be affected by several factors such as the presence of infection, previous periodontal disease, the extent of a traumatic injury and the number or the thickness of the bony socket walls (Garg 2001). An equilibrium is reached approximately $3-4$ months post-extraction, resulting in a bone and soft tissue level that is lower than that of the neighbouring teeth as complete regeneration of the socket site never occurs (Amler 1969).

To reduce the loss of alveolar bone to an acceptable level, several alveolar ridge preservation (ARP) techniques' procedures have been proposed (Wang et al. 2004; Horvath et al. 2013). These have included the minimally traumatic extraction of a tooth, followed by immediate grafting of the extraction sockets using particulate bone grafts or substitutes, guided bone regeneration (GBR) with or without bone grafts or substitutes (Adriaens \& Van Stede 1998; Iasella et al. 2003; Mardas et al. 2010) and a socket seal technique using different tissue graft materials (Lekovic et al. 1998; Bartee 2001; Jung et al. 2004; Araujo et al. 2015). The use of grafting materials as an adjunct to GBR or socket seal techniques is based on the assumption that this material may be useful not only in prohibiting membrane or soft tissue graft collapse into the socket area but also in enhancing new bone formation through osteoinduction and osteoconduction.

Direct grafting and augmentation of the extraction socket has been proposed using autogenous bone (Becker et al. 1994), demineralised freeze-dried bone allograft (Becker et al. 1994; Becker et al. 1996; Froum et al. 2002), mineralised freeze-dried bone allograft (Feuille et al. 2003a,b), deproteinised bovine bone (Artzi et al. 2000 and Mardas et al. 2010), alloplastic polymers (Gross 1995 and Serino et al. 2003), bioactive glasses (Froum et al. 2002) and composite ceramic materials (Mardas et al. 2010). Although these bone substitutes were able to maintain the tissue contours in extraction sites, the conservancy of the gingival and bone tissue was variable. Marked differences in the quantity and the quality of the regenerated tissue have been reported, with the presence of the graft sometimes identified as interfering with the normal healing process (Froum et al. 2002; Mardas et al. 2010; Horvath et al. 2013; Hsun-Liang et al. 2013).

The preservation and regeneration of the gingival tissue is also important as it helps to establish an ideal functional and aesthetic foundation, before prosthetic rehabilitation occurs (Seibert 1983; Studer et al. 2000; Jung et al. 2004; Prato et al. 2004). Although there is recognition that various ARP techniques can be used to preserve and promote alveolar bone and soft tissue development in the extraction socket area (Vignoletti et al. 2012; Wang \& Lang 2012; De Risi et al. 2013; Horvath et al. 2013; Avila-Ortiz et al. 2014; Morjaria et al. 2014), heterogeneity of the published data has led Vignoletti et al. (2012), Horvath et al. (2013), Mardas et al. (2015) to conclude that the clinical outcome and prosthetic options available following ARP are inconclusive.

This systematic review and meta-analysis have been designed to investigate the effects of alveolar ridge preservation on bone and gingival tissue site dimensions, keratinised tissue width, histological bone characteristics and patient-based outcomes. It was designed as an extension and update of the systematic reviews undertaken by Horvath et al. (2013) Mardas et al. (2015).

\section{Material and methods}

A detailed protocol was developed based on the design of a previous systematic review undertaken as a component of the 4th EAO consensus. This study reviewed therapeutic concepts for improving dental implant outcomes following tooth extraction (Mardas et al. 2015).

\section{Focused question 1}

The main focused question of this systematic review was as follows: "Is there any additional benefit of alveolar ridge preservation techniques over unassisted healing in terms of the following: (i) horizontal and vertical alveolar ridge dimensions, (ii) soft tissue conservancy measured through linear and volumetric analysis, (iii) histological characteristics of the bone, (iv) keratinised tissue dimensions and (V) patient-based outcomes?

\section{Focused question 2}

This question was designed to examine data published in case series and in controlled clinical studies, where unassisted socket healing had not been used as a control group. It aimed to examine the effects of different ARP procedures and to address a second focused question: "what are the estimated size effects on (i) horizontal and vertical alveolar ridge dimensions, (ii) gingival tissue conservancy measured through linear and volumetric dimensional changes, (iii) histological characteristics of the bone, (iv) keratinised tissue dimensions and (V) patientbased outcomes, following different alveolar ridge preservation techniques?"

\section{Types of studies}

For focused question 1, only longitudinal prospective studies, that is RCTs and CCTs with unassisted socket healing as a control group, were included in the meta-analysis.

For focused question 2, in addition to the previous studies, RCTs, CCTs and large prospective case series without an unassisted healing control group were included in the meta-analysis.

\section{Populations of studies}

Healthy individuals, without any age limit, underwent any type of ARP following permanent tooth extraction. Studies including smokers and patients with a history of periodontal disease were not excluded.

\section{Types of interventions}

Test group

Studies reporting on any of the following ARP interventions were included: (i) socket grafting with autographs, allografts, xenografts, alloplast and substitutes with biologically active materials (growth factors); (ii) GBR with various barrier membranes and combinations of the above grafting materials; and (iii) socket seal procedures using a combination of soft tissue graft and the above grafting materials.

\section{Control group}

The control group for focused question 1 was unassisted socket healing following atraumatic tooth extraction without any other intervention.

\section{Outcome variables}

For both focused questions, the following outcome variables were evaluated:

1. Linear and/or volumetric changes in vertical alveolar bone height.

2. Linear and/or volumetric changes in alveolar bone width.

3. Soft tissue dimensional changes.

4. Histological characteristics of new bone formation.

5. Changes in keratinised tissue width and thickness.

6. Post-operative complications and patientbased outcomes. 
Risk of bias and methodological quality assessment

An assessment of the risk of bias within the study and the research methodology was undertaken using a modification of the Cochrane tool proposed by Higgins \& Green (2011). No attempt was made to differentiate between non-randomised and randomised studies as both randomised and non-randomised clinical trials were included in the systematic review. The levels of bias were classified as low risk, unclear risk or high risk, with six parameters: allocation concealment, blinding of participants and personnel, blinding of outcome assessment, incomplete outcome data, selective reporting and other sources of bias evaluated. If all the parameters were judged as low, the study was at low risk of bias. If at least one parameter was judged as unclear or as at high risk of bias, the studies were included at unclear or high risk of bias, respectively.

\section{General inclusion criteria}

1. Studies on healthy individuals, without any age limit, who underwent ARP following tooth extraction.

2. Studies providing information on bone and soft tissue characteristics and patient-based outcomes following ARP at an extraction socket site.

\section{General exclusion criteria}

1. Retrospective studies.

2. Studies on medically compromised patients or under specific medication.

3. Studies reporting on immediate implant placement as a method for ARP.

4. Studies reporting solely on third molar extractions.

5. Publications reporting data on the same sample and procedures as other publications.

Specific inclusion criteria for focused question 1

1. Longitudinal prospective studies, that is RCTs and CCTs where one of the abovementioned types of interventions was carried out in the test group and where unassisted socket healing was used as a control group.

2. Studies reporting on a minimum of 10 patients per group.

3. Follow-up time longer than 3 months.

Specific inclusion criteria for focused question 2

1. Longitudinal prospective studies, that is RCTs, CCTs, cohort studies where one or more of the above-mentioned types of interventions was carried out, with or without unassisted socket healing as a control group, and prospective case series.

2. Controlled studies reporting on a minimum of 10 patients per group, or case series reporting on a minimum of 20 patients.

3. Follow-up time longer than 3 months.

\section{Search strategy}

The search strategy incorporated both electronic search and hand-search. The following electronic databases were utilised: (i) MEDLINE In-Process \& Other Non-Indexed Citations and MEDLINE 1950 to present via Ovid interface; (ii) EMBASE Classic + EMBASE 1947 to present via Ovid interface; (iii) The Cochrane Central Register of Controlled Trials (CENTRAL); (iv) LILACS; and (v) Web of Science. The electronic search strategy included terms related to the intervention and used the following combination of keywords and MeSH terms: ("tooth extraction" OR "tooth removal" OR "socket" OR "alveol" OR "ridge" OR "crest" OR "toothsocket" OR "alveolarboneloss" OR "boneresorption" OR "boneremodelling"|AND ("preserv" OR "reconstruct" OR "augment" OR "fill" OR "seal" OR "graft" OR "repair" OR "alveolar ridge augmentation" OR "bone regeneration" OR "bone substitutes" OR "transplantation"). Cochrane search filters for RCTs and CCTS were implemented, with cohort trials also included. The results were limited to human studies. The full electronic search strategy can be found in the Appendix. An extensive hand-search was also performed encompassing the bibliographies of the included papers and other narrative and systematic reviews. In addition, the following journals were screened from 2001 to July 2014: Clinical Oral Implants Research, Clinical Implant Dentistry and Related Research, European Journal of Oral Implantology, Implant Dentistry, International Journal of Oral and Maxillofacial Implants, International Journal of Periodontics and Restorative Dentistry, Journal of Clinical Periodontology, Journal of Dental Research, Journal of Oral and Maxillofacial Surgery, Journal of Periodontology, Oral Surgery, Oral Medicine, Oral Radiology, Oral Pathology and Endodontics. No language restrictions were applied, and translations were carried out if necessary. Unpublished trials and abstracts were not included in the search process. When the results of a study were presented in a number of publications, the most complete data set was included in the analysis. In case of missing or incomplete data, the authors were contacted via email allowing a period of 3 weeks for their reply with the missing data. The extracted data were copied into EndNote X7 software (Thomson Reuters, New York, NY, USA), and all further steps of screening were performed on this interface. A three-stage selection of the resulted hits was performed independently and in duplicate by two reviewers (ATE and NDM). In order to reduce errors and bias, a calibration exercise was performed with the first 24 articles identified from the journal hand-search. In case of disagreement at the title selection stage, the trial was included in the abstract stage. At the abstract and full-text selection, any disagreements between the above reviewers were resolved by discussion including a third reviewer (NM). The reasons for exclusion were recorded in a specific data extraction form at the full-text selection stage. The level of agreement was determined by a kappa score calculation of agreement during the title and abstract selection process.

\section{Research synthesis and meta-analysis}

For all included studies answering both focused questions, a descriptive synthesis was undertaken. The studies were classified according to research design and type of intervention and the outcomes were recorded in evidence tables.

For focused question 1, meta-analysis was conducted utilising the available data from the selected RCT and CCT studies. The analysis was undertaken separating the studies according to parallel and split-mouth designs and was only carried out if each group contained more than 2 eligible studies.

For focused question 2, meta-analysis was conducted utilising the available data from all the studies included in the analysis of focused question 1 and data from RCTs and CCTs with parallel design, as well as larger prospective case series. The studies included for meta-analysis were divided into three different groups $\mid G B R$, socket grafting and socket seal) with analysis only carried out if each group contained more than 2 eligible studies. When ARP was performed utilising a resorbable or non-resorbable barrier membrane, the study was categorised in the $G B R$ group. This was independent of whether an additional bone grafting material was used. When the socket was treated with a bone or substitute graft, including collagen sponges/ plaques and growth factors, the study was categorised in the socket grafting group. Finally, the study was categorised in the 
socket seal group when a soft tissue graft was used to seal the entrance of the socket with or without grafting of the socket following a flapless approach.

MedCalc ${ }^{\circledR}$ version 15.11.0 (MedCalc Software bvba, Ostend, Belgium) software was used for the meta-analyses for focused question 1. For question 2, Comprehensive Meta Analysis version 3.3.070 (Biostat, Inc., Englewood, NJ, USA) software was used.

When several intervention groups were reported on, these were combined into one single intervention group, as advised in The Cochrane Handbook for Systematic Reviews of Interventions (Higgins \& Green 2011).

Assessment of statistical heterogeneity was performed using Cochran's Q-test and determination of the $I^{2}$ index (Higgins et al. 2003). The $I^{2}$ index provides an estimate of the amount of variation attributable to heterogeneity $\left(I^{2}=25 \%\right.$ : low; $I^{2}=50 \%$ : moderate; $I^{2}=75 \%$ : high heterogeneity). The different outcome variable estimates were pooled using a random effects model, as the effect of ARP was anticipated as varying between individual studies (Borenstein et al. 2009).

For questions 1 and 2, a standardised mean difference (i.e. the difference in means divided by the standard deviation) was calculated for continuous variables. For question 2 , forest plots were created to illustrate the effects of the different studies, shown against the global estimate.

Statistical significance was achieved if $P<0.05$. The unit of analysis used for the study was the patient. Results are given as mean \pm standard deviation (SD) unless stated differently.

\section{Results}

\section{Study selection}

The initial search yielded a total 14,409 records including 82 papers that were selected through hand-search and two more through cross-reference. After removal of duplicates and title and abstract screening, a total of 112 articles were left for full-text assessment (Fig. 1). The authors of 5 of these 112 articles were contacted at this stage to provide additional data on ARP dimensional outcomes before the final selection.

The most common reason for exclusion of papers was insufficient numbers of patient, no relevant outcome data, data which was relevant but recorded in a manner/format which was incompatible with the inclusion criteria, duplicate report, insufficient followup time and the study design not matching research protocol. The excluded papers and (a)

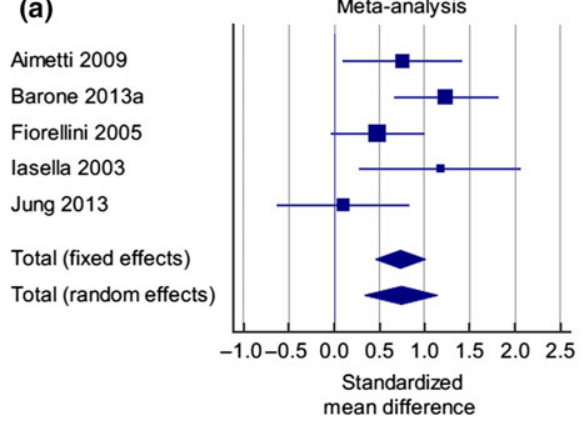

(c)

\section{Aimetti 2009}

Barone 2013a

lasella 2003

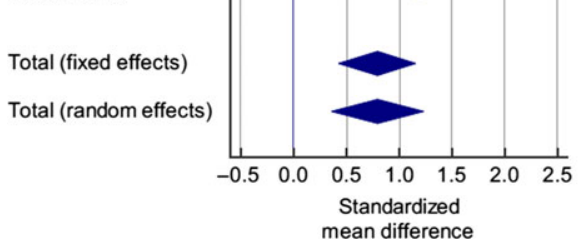

(b)

Camargo 2000

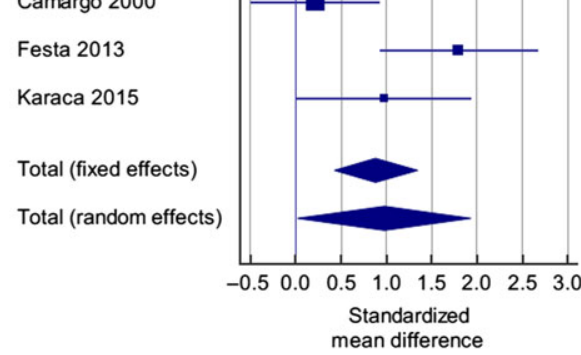

(d)

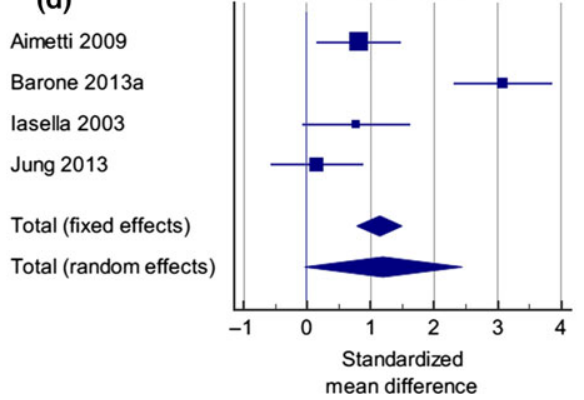

Fig. 1. Meta-analysis results and heterogeneity test for Q1; parallel studies, (a) Parallel studies investigating linear and volumetric changes in vertical alveolar bone height (Mid-Buccal). (b) Split mouth studies reporting on changes in the mid-buccal vertical alveolar ridge dimensions. (c) Parallel studies investigating linear and volumetric changes in vertical alveolar bone height (proximal). (d) Parallel studies investigating linear and volumetric changes in alveolar bone width.

the reasons for exclusion for both focused questions are listed in Table 1.

The kappa score for agreement between the reviewers (ATE and NDM) at the title and abstract selection level was 0.95 , indicating a high level of agreement.

\section{Study design and population}

The study design and study population characteristics of the included studies for both focused questions are presented in Table 2.

Controlled studies answering the focus question 1 Nine papers (Camargo et al. 2000; Iasella et al. 2003; Fiorellini et al. 2005; Aimetti et al. 2009; Barone et al. 2013a; Festa et al. 2013; Jung et al. 2013; Cardaropoli et al. 2014; Karaca et al. 2015) were eligible for inclusion in the qualitative analysis for focused question 1. Eight of the studies (Iasella et al. 2003; Fiorellini et al. 2005; Aimetti et al. 2009; Barone et al. 2013a; Festa et al. 2013; Jung et al. 2013; Cardaropoli et al. 2014; Karaca et al. 2015) were designed as RCT trials, with one (Camargo et al. 2000) a CCT. Six of the studies were of a parallel design (Iasella et al. 2003; Fiorellini et al. 2005; Aimetti et al. 2009; Barone et al. 2013a; Jung et al. 2013; Cardaropoli et al. 2014) and three studies (Camargo et al. 2000; Festa et al. 2013; Karaca et al. 2015) of a split-mouth design. Five of the studies
(Camargo et al. 2000; Fiorellini et al. 2005; Aimetti et al. 2009; Festa et al. 2013; Jung et al. 2013) performed ARP utilising socket grafting procedures, three studies used GBR (Iasella et al. 2003; Barone et al. 2013a; Cardaropoli et al. 2014), and one study used socket sealing (Karaca et al. 2015).

Follow-up after ARP ranged from 3 to 6 months. Two studies (Aimetti et al. 2009; Karaca et al. 2015) measured the dimensions of the post-extraction alveolar ridge at 3 months, three (Fiorellini et al. 2005; Barone et al. 2013a; Cardaropoli et al. 2014) at 4 months, one at 4 and 6 months (Iasella et al. 2003) and the remaining three (Camargo et al. 2000; Festa et al. 2013; Jung et al. 2013) at 6 months.

All of the included studies measured alveolar and gingival tissue site dimensions using direct intraoral measurements /Camargo et al. 2000; Iasella et al. 2003; Aimetti et al. 2009; Barone et al. 2013a; Festa et al. 2013; Cardaropoli et al. 2014) or radiographic CBCT analysis (Fiorellini et al. 2005, Jung et al. 2013; Karaca et al. 2015).

Eight (Camargo et al. 2000; Iasella et al. 2003; Fiorellini et al. 2005; Aimetti et al. 2009; Festa et al. 2013; Jung et al. 2013; Cardaropoli et al. 2014; Karaca et al. 2015) of the nine included studies prescribed pre- or postoperative antibiotics. 
Table 1. List of excluded full-text papers and reasons for exclusion following full-text screening

\begin{tabular}{|c|c|}
\hline Author and year & Reasons for exclusion \\
\hline Alkan 2013 & Insufficient number of patients \\
\hline Al-Khaldi 2011 & No relevant outcome data or data provided in incompatible format \\
\hline Anitua 1999 & Insufficient number of patients \\
\hline Anitua 2015 & $\begin{array}{l}\text { No relevant outcome data or data provided in incompatible format, insufficient } \\
\text { follow-up }\end{array}$ \\
\hline Araujo 2015 & No relevant outcome data or data provided in incompatible format \\
\hline Artzi 2000 & Insufficient number of patients \\
\hline Babbush 2003 & Insufficient number of patients \\
\hline Barone 2008 & Duplicate report (Barone 2012) \\
\hline Barone 2012 & No relevant outcome data or data provided in incompatible format \\
\hline Brkovic 2012 & Insufficient number of patients \\
\hline $\begin{array}{l}\text { Brownfield } \\
2012\end{array}$ & Insufficient number of patients \\
\hline Canullo 2013 & Insufficient number of patients \\
\hline Canuto 2013 & $\begin{array}{l}\text { No relevant outcome data or data provided in incompatible format, insufficient } \\
\text { follow-up }\end{array}$ \\
\hline $\begin{array}{l}\text { Cardaropoli } \\
2012\end{array}$ & Duplicate report (Cardaropoli 2014) \\
\hline $\begin{array}{l}\text { Carmagnola } \\
2003\end{array}$ & Insufficient number of patients \\
\hline Casado 2010 & Insufficient number of patients \\
\hline Crespi 2009 & No relevant outcome data or data provided in incompatible format \\
\hline Clozza 2012 & Duplicate report (Clozza 2014), insufficient number of patients \\
\hline Clozza 2014 & Insufficient number of patients \\
\hline Collins 2014 & Insufficient number of patients \\
\hline De Coster 2011 & Insufficient number of patients, study seems to be retrospective \\
\hline $\begin{array}{l}\text { Engler-Hamm } \\
2011\end{array}$ & Insufficient number of patients \\
\hline Farina 2013 & No relevant outcome data or data provided in incompatible format \\
\hline Fotek 2009 & Insufficient number of patients \\
\hline Flugge 2015 & Unclear study design \\
\hline Geffre 2010 & Animal study \\
\hline Geurs 2014 & Insufficient number of patients \\
\hline Hanser 2014 & Study seems to be retrospective \\
\hline Hauser 2013 & Insufficient number of patients \\
\hline Heberer 2008 & Insufficient number of patients \\
\hline Heberer 2011 & Insufficient number of patients \\
\hline Heberer 2012 & Insufficient number of patients \\
\hline $\begin{array}{l}\text { Hernández- } \\
\text { Alfaro } 2005\end{array}$ & $\begin{array}{l}\text { Insufficient number of patients, reports on a mixture of clinical situations } \\
\text { (ARP, discrepancy implant socket, reconstruction after removal of implants, etc.) }\end{array}$ \\
\hline Hsuan-Yu 2012 & Insufficient number of patients \\
\hline Irinakis 2006 & Review article \\
\hline Jung 2004 & $\begin{array}{l}\text { No relevant outcome data or data provided in incompatible format, insufficient } \\
\text { follow-up }\end{array}$ \\
\hline Kim 2011 & No relevant outcome data or data provided in incompatible format \\
\hline Kim 2013 & Insufficient number of patients \\
\hline Kotsakis $2014 a$ & Insufficient number of patients \\
\hline Kotsakis 2014b & Insufficient number of patients \\
\hline Lambert 2012 & Insufficient number of patients \\
\hline $\begin{array}{l}\text { Leblebicioglu } \\
2013\end{array}$ & No relevant outcome data or data provided in incompatible format \\
\hline Lekovic 1998 & Duplicate report (Camargo et al. 2000) \\
\hline Luczyszyn 2005 & Insufficient number of patients \\
\hline Madan 2014 & Insufficient number of patients \\
\hline Mahesh 2012 & Study design \\
\hline Mardas 2011 & Duplicate report (Mardas et al. 2010) \\
\hline $\begin{array}{l}\text { Mardinger } \\
2009\end{array}$ & Duplicate report (Mardinger et al. 2012) \\
\hline Misch 2010 & Insufficient number of patients \\
\hline $\begin{array}{l}\text { Moghaddas } \\
2012\end{array}$ & Insufficient number of patients \\
\hline Nam 2011 & No relevant outcome data or data provided in incompatible format \\
\hline Neiva 2011 & Insufficient number of patients \\
\hline Ntounis 2015 & Insufficient follow-up \\
\hline Nevins 2014 & Insufficient number of patients \\
\hline Norton 2002 & Insufficient number of patients \\
\hline Oghli 2010 & No relevant outcome data or data provided in incompatible format \\
\hline Patel 2013 & Duplicate (Mardas et al. 2010) \\
\hline Pellegrini 2014 & Insufficient number of patients \\
\hline Ruga 2011 & Insufficient number of patients \\
\hline Scheyer 2012 & Insufficient number of patients \\
\hline Schneider 2014 & Duplicate report (Jung et al. 2013) \\
\hline Serino 2008 & Insufficient number of patients \\
\hline
\end{tabular}

Five parallel studies (Iasella et al. 2003; Fiorellini et al. 2005; Aimetti et al. 2009; Barone et al. 2013a; Jung et al. 2013) were included in the meta-analysis. Cardaropoli et al. (2014) was excluded from the meta-analysis as the study used the socket as the unit of analysis, preventing pooling of data. A separate meta-analysis was carried out for the split-mouth studies undertaken by Festa et al. (2013), Camargo et al. (2000); Karaca et al. (2015).

The study population ranged from 15 to 80 patients in the included studies. This resulted in 194 patients being considered in the meta-analysis. One hundred and fiftythree patients were present in parallel studies and 41 in the split-mouth studies. The distribution of the extracted teeth included both single- and multi-rooted teeth. Two of the studies included smokers (Barone et al. 2013a; Jung et al. 2013), two studies (Aimetti et al. 2009; Festa et al. 2013) excluded smokers and four (Camargo et al. 2000; Fiorellini et al. 2005; Iasella et al. 2003; Karaca et al. 2015) did not report on smoking habits.

Studies answering the focus question 2

Thirty-seven studies (Camargo et al. 2000; Iasella et al. 2003; Serino et al. 2003; Vance et al. 2004; Fiorellini et al. 2005; Pinho et al. 2006; Neiva et al. 2008; Aimetti et al. 2009; Crespi et al. 2009, 2011a,b; Beck \& Mealey 2015; Borg and Mealey 2010; Mardas et al. 2010; Fernandes et al. 2011; Huh et al. 2011; Gholami et al. 2012; Hoang \& Mealey 2012; Mardinger et al. 2012; Perelman-Karmon et al. 2012; Wood \& Mealey 2012; Barone et al. 2013a,b, 2014; Cook \& Mealey 2013; Festa et al. 2013; Jung et al. 2013; Poulias et al. 2013; Wallace et al. 2013; CalasansMaia et al. 2014; Cardaropoli et al. 2014; Coomes et al. 2014; Eskow \& Mealey 2014; Kim et al. 2014; Lindhe et al. 2014; Karaca et al. 2015; Meloni et al. 2015) were included in the qualitative analysis of question 2. Twenty-nine studies (Iasella et al. 2003; Vance et al. 2004; Fiorellini et al. 2005; Pinho et al. 2006; Neiva et al. 2008; Aimetti et al. 2009; Borg and Mealey 2010; Mardas et al. 2010; Crespi et al. 2011b; Fernandes et al. 2011; Huh et al. 2011; Gholami et al. 2012; Hoang \& Mealey 2012; Perelman-Karmon et al. 2012; Wood \& Mealey 2012; Barone et al. 2013a,b, 2014; Cook \& Mealey 2013; Festa et al. 2013; Jung et al. 2013; Poulias et al. 2013; Calasans-Maia et al. 2014; Cardaropoli et al. 2014; Coomes et al. 2014; Eskow \& Mealey 2014; Kim et al. 2014; Karaca et al. 2015; Meloni et al. 2015) were 
Table 1. (continued)

\begin{tabular}{ll}
\hline Author and year & Reasons for exclusion \\
\hline Simon 2011 & No relevant outcome data or data provided in incompatible format \\
Sisti 2012 & Insufficient follow-up \\
Shakibaie 2013 & Insufficient number of patients \\
Spinato 2014 & No relevant outcome data or data provided in incompatible format \\
Suttapreyasri 2013 & Insufficient number of patients \\
Tal 1999 & Unclear study design and insufficient follow-up \\
Tete 2013 & Reports on a mixture of clinical situations (ARP vs. sinus augmentation), \\
& insufficient follow-up \\
Thalmair 2013 & Insufficient number of patients \\
Toloue 2012 & Unclear study design \\
Vanhoutte 2014 & Duplicate report (Lambert 2012) \\
Villanueva- & Insufficient number of patients \\
Alcojol 2013 & Insufficient number of patients \\
Weiss 2007 & Insufficient number of patients \\
Wu 2014 &
\end{tabular}

designed as a RCT, seven studies /Camargo et al. 2000; Serino et al. 2003; Crespi et al. 2009, 2011a; Beck \& Mealey 2010; Wallace et al. 2013; Lindhe et al. 2014) designed as a CCT and one study (Mardinger et al. 2012) was a prospective case series.

Eleven studies (Fiorellini et al. 2005; Neiva et al. 2008; Beck \& Mealey 2010; Crespi et al. 2011a; Huh et al. 2011; Hoang \& Mealey 2012; Wood \& Mealey 2012; Jung et al. 2013; Calasans-Maia et al. 2014; Coomes et al. 2014 and Eskow \& Mealey 2014) compared two different grafting techniques with seven studies (Iasella et al. 2003; Serino et al. 2003; Aimetti et al. 2009; Crespi et al. 2009, 2011b; Festa et al. 2013; Cardaropoli et al. 2014) comparing a grafting procedure with unassisted socket healing. One study (Barone et al. 2013a) compared GBR with unassisted socket healing, twelve studies (Pinho et al. 2006; Mardas et al. 2010; Fernandes et al. 2011; Gholami et al. 2012; Perelman-Karmon et al. 2012; Barone et al. 2013b, 2014; Cook \& Mealey 2013; Poulias et al. 2013; Wallace et al. 2013; Kim et al. 2014; Borg \& Mealey 2015) compared different GBR techniques. Four studies (Mardinger et al. 2012; Lindhe et al. 2014; Karaca et al. 2015; Meloni et al. 2015) compared different socket seal techniques, and one study (Vance et al. 2004) compared a grafting procedure against GBR Finally, 3 studies (Fiorellini et al. 2005; Crespi et al. 2009; Jung et al. 2013) compared multiple grafting techniques against an unassisted healing control.

Follow-up times ranged from 3 to 9 months after the ARP. Seven studies (Neiva et al. 2008; Aimetti et al. 2009; Crespi et al. 2009; Huh et al. 2011; Barone et al. 2014; Kim et al. 2014; Karaca et al. 2015) examined dimensions after 3 months of healing, 16 studies after 4-6 months (Iasella et al. 2003; Poulias et al. 2013; Vance et al. 2004; Fiorellini et al. 2005; Beck \& Mealey 2010;
Crespi et al. 2011a,b; Wood \& Mealey 2012; Barone et al. 2013a; Cook \& Mealey 2013; Wallace et al. 2013; Cardaropoli et al. 2014; Coomes et al. 2014; Eskow \& Mealey 2014; Borg \& Mealey 2015; Meloni et al. 2015), 13 studies after 6-9 months (Camargo et al. 2000; Serino et al. 2003; Pinho et al. 2006; Mardas et al. 2010; Fernandes et al. 2011; Gholami et al. 2012; Hoang \& Mealey 2012; Mardinger et al. 2012; Barone et al. 2013b; Festa et al. 2013; Jung et al. 2013; CalasansMaia et al. 2014; Lindhe et al. 2014) and one study after 9 months (Perelman-Karmon et al. 2012).

Twenty-eight of the studies measured alterations in site dimensions. Twenty-two (Camargo et al. 2000; Iasella et al. 2003; Serino et al. 2003; Vance et al. 2004; Pinho et al. 2006; Neiva et al. 2008; Aimetti et al. 2009; Beck \& Mealey 2010; Borg and Mealey 2010; Mardas et al. 2010; Fernandes et al. 2011; Gholami et al. 2012; Hoang \& Mealey 2012; Wood \& Mealey 2012; Barone et al. 2013a, 2014; Cook \& Mealey 2013; Festa et al. 2013; Poulias et al. 2013; Calasans-Maia et al. 2014; Cardaropoli et al. 2014; Eskow \& Mealey 2014; Karaca et al. 2015) directly measured the alteration in the size of alveolar complex, with seven studies recording measurements from intraoral (Crespi et al. 2009) or CBCT (Fiorellini et al. 2005; Huh et al. 2011; Jung et al. 2013; Kim et al. 2014; Karaca et al. 2015; Meloni et al. 2015) radiographic images. One study measured both intraoral and radiographic measurements (Coomes et al. 2014). Seven studies (Crespi et al. 2011a,b; Mardinger et al. 2012; Perelman-Karmon et al. 2012; Barone et al. 2013b; Wallace et al. 2013; Lindhe et al. 2014) did not attempt to measure dimensional changes of the hard tissues but provided either histological information or soft tissue changes.

Twenty-nine (Camargo et al. 2000; Iasella et al. 2003; Vance et al. 2004; Pinho et al.
2006; Neiva et al. 2008; Aimetti et al. 2009; Crespi et al. 2009, 2011a,b; Beck \& Mealey 2010; Mardas et al. 2010; Fernandes et al. 2011; Gholami et al. 2012; Hoang \& Mealey 2012; Mardinger et al. 2012; Wood \& Mealey 2012; Cook \& Mealey 2013; Festa et al. 2013; Jung et al. 2013; Barone et al. 2014; CalasansMaia et al. 2014; Cardaropoli et al. 2014; Coomes et al. 2014; Eskow \& Mealey 2014; Kim et al. 2014; Lindhe et al. 2014; Borg \& Mealey 2015; Karaca et al. 2015) of the 37 included studies prescribed pre- or post-operative antibiotics. Four studies (Perelman-Karmon et al. 2012; Barone et al. 2013a; Poulias et al. 2013 - one group and Serino et al. 2003) did not prescribe $A B$ as a component of treatment, and five studies (Neiva et al. 2008; Huh et al. 2011; Barone et al. 2013b; Poulias et al. 2013 - one group and Wallace et al. 2013) did not provide this information.

Eighteen studies were included in the meta-analysis (Iasella et al. 2003; Vance et al. 2004; Fiorellini et al. 2005; Neiva et al. 2008; Aimetti et al. 2009; Mardas et al. 2010; Huh et al. 2011; Hoang \& Mealey 2012; Wood \& Mealey 2012; Barone et al. 2013a, 2014; Jung et al. 2013; Poulias et al. 2013; CalasansMaia et al. 2014; Coomes et al. 2014; Kim et al. 2014; Borg \& Mealey 2015; Meloni et al. 2015). The study population ranged from 20 to 80 patients. Following categorisation into intervention groups, 266 patients were considered for the meta-analysis of the GBR group, 317 patients for the meta-analysis of the socket grafting group and 50 patients for the meta-analysis of the socket seal group. Although the distribution of the teeth extracted in the GBR and socket grafting groups was fairly heterogeneous and included both single- and multi-rooted teeth, the location of the extracted teeth in the socket seal group was mainly maxillary, nonmolar teeth.

Seven of the studies included both smokers and non-smokers (Mardas et al. 2010; Barone et al. 2013a, 2014; Jung et al. 2013; Poulias et al. 2013; Coomes et al. 2014; Meloni et al. 2015), six (Iasella et al. 2003; Vance et al. 2004; Fiorellini et al. 2005; Huh et al. 2011; Hoang \& Mealey 2012; Kim et al. 2014) did not report on smoking habits, and five studies (Neiva et al. 2008; Aimetti et al. 2009; Wood and Mealey 2012; Calasans-Maia et al. 2014; Borg \& Mealey 2015) excluded smokers.

\section{Intervention characteristics}

The interventional characteristics of the included studies for both focused questions are presented in Table 2. 
Controlled studies answering the focused question 1 In four of the nine included studies /Camargo et al. 2000; Iasella et al. 2003; Fiorellini et al. 2005; Festa et al. 2013), mucoperiosteal flaps were elevated at both the ARP-treated and control extraction sites. In one paper (Cardaropoli et al. 2014), a flap was only raised in the treatment group. In the remaining four studies (Aimetti et al. 2009; Barone et al. 2013a,b; Jung et al. 2013; Karaca et al. 2015), a flapless approach was followed. Primary closure was attempted in both the treatment and control groups in one study (Fiorellini et al. 2005), with one study (Jung et al. 2013) undertaking primary closure in two of three treatment groups and one study (Festa et al. 2013) only in the control group. In the five studies that specified the number of intact walls at the extraction site, all had at least 3 walls intact after extraction of the tooth (Fiorellini et al. 2005; Aimetti et al. 2009; Festa et al. 2013; Jung et al. 2013; Cardaropoli et al. 2014).

In three of the nine included studies, ARP was performed using a collagen barrier for GBR in combination with a porcine xenograft (Barone et al. 2013a; Cardaropoli et al. 2014) or an allograft (Iasella et al. 2003). In three studies, socket grafting was undertaken using an alloplast material, with calcium sulphate or calcium phosphate (Aimetti et al. 2009; Jung et al. 2013) and bioactive glass (Camargo et al. 2000) utilised. In one study, a porcine xenograft with a porcine cortical layer was used for grafting of the sockets (Festa et al. 2013), and in another study, a polylactide-polyglycolide acid sponge and human BMP was provided (Fiorellini et al. 2005). Two socket seal techniques were examined against a socket grafting technique in one study (Jung et al. 2013), with the effects of a porcine collagen matrix seal compared against a connective tissue graft. One study (Karaca et al. 2015) examined the effects of socket sealing using a free gingival graft.

\section{Studies answering the focused question 2}

\section{$G B R$}

In seven of the ten included studies (Iasella et al. 2003; Vance et al. 2004; Pinho et al. 2006; Mardas et al. 2010; Fernandes et al. 2011; Poulias et al. 2013; Kim et al. 2014; Borg \& Mealey 2015), mucoperiosteal flaps were elevated as a component of the surgery. Two studies adopted a flapless surgical technique (Barone et al. 2013a, 2014 - one group). Pinho et al. (2006) and Barone et al. (2014 one group) attempted primary closure at the tooth extraction site following GBR augmentation.

In the four studies that specified the number of intact walls required for inclusion in the study, all had at least 3 walls of the socket walls remaining intact, with greater that $50 \%$ of the 4 th wall remaining after extraction of the tooth (Mardas et al. 2010; Barone et al. 2014; Kim et al. 2014; Borg \& Mealey 2015).

$G B R$ was performed in most of the studies using a collagen barrier in combination with either a porcine or bovine xenograft (Barone et al. 2013a, 2014; Mardas et al. 2010 - one group and Vance et al. 2004), hydroxyapatite (Cook \& Mealey 2013 - one group, Gholami et al. 2012 - one group), synthetic ceramic (Mardas et al. 2010 - one group) or freezedried bone allograft (-PTFE membrane, Iasella et al. 2003; Vance et al. 2004 - one group; Poulias et al. 2013; Kim et al. 2014; Borg \& Mealey 2015). One study (Pinho et al. 2006) used an autograft harvested from the maxillary tuberosity in combination with a titanium barrier. An acellular dermal matrix barrier in combination with an acellular dermal matrix allograft was used by Fernandes et al. (2011), and a resorbable polylactide barrier with cancellous allograft with or without bovine xenograft was used by Poulias et al. (2013).

\section{Socket grafting}

In five of the twelve included studies (Camargo et al. 2000; Vance et al. 2004; Fiorellini et al. 2005; Festa et al. 2013; CalasansMaia et al. 2014), mucoperiosteal flaps were elevated as a component of the surgery. Four studies adopted a flapless surgical technique (Neiva et al. 2008; Aimetti et al. 2009; Jung et al. 2013; Coomes et al. 2014). It was unclear whether flaps were elevated in three studies (Huh et al. 2011; Hoang \& Mealey 2012; Wood \& Mealey 2012). Primary tissue closure was attempted in four of the studies /Calasans-Maia et al. 2014; Fiorellini et al. 2005; Jung et al. 2013 - two groups and Festa et al. 2013 - one group), with only one group in one study not specifying the surgical technique (Huh et al. 2011). All other groups did not attempt primary closure.

In the eight studies that specified the number of intact walls required for inclusion in the study, all required at least 3 walls of the socket wall remaining intact, with greater that $50 \%$ of the fourth wall remaining after extraction of the tooth (Fiorellini et al. 2005; Neiva et al. 2008; Aimetti et al. 2009; Hoang \& Mealey 2012; Festa et al. 2013; Jung et al. 2013; Coomes et al. 2014).
Socket grafting was performed using either allografts (Calasans-Maia et al. 2014), xenografts (Vance et al. 2004 - one group; Festa et al. 2013), xenografts combined with a synthetic collagen peptide collagen known as P15 (Neiva et al. 2008 - one group), alloplasts and bioactive glass materials /Camargo et al. 2000; Aimetti et al. 2009; Jung et al. 2013 one group), a combination of alloplasts, xenografts and rhBMP-2 (Huh et al. 2011), a combination of allograft and collagen (Wood \& Mealey 2012), a combination of synthetic polymer, ceramic material and allograft (Vance et al. 2004 - one group), a demineralised xenograft matrix in bovine collagen and sodium alginate carrier (Hoang \& Mealey 2012) and a collagen carrier with and without rhBMP-2 (Fiorellini et al. 2005; Coomes et al. 2014).

\section{Socket seal}

All three included studies (Jung et al. 2013; Karaca et al. 2015; Meloni et al. 2015) adopted a flapless surgical technique. Two of these studies required patients to have at least 3 walls of the socket walls intact, with the fourth wall having greater than $50 \%$ of the buccal bone remaining or a dehiscence or fenestration of $<3 \mathrm{~mm}$. No description of the socket wall morphology was provided by Karaca et al. (2015).

Both Meloni et al. (2015), Jung et al. (2013) examined the effects of socket sealing using a bone allograft and either a connective tissue (Meloni et al. 2015) or free gingival graft (Jung et al. 2013) in comparison with ARP using an allograft and porcine collagen matrix. The allograft in the Jung et al. (2013) study was a deproteinised bovine bone mineral with $10 \%$ collagen. Karaca et al. (2015) examined the isolated effect of using a free gingival graft for socket sealing.

\section{Outcome variables}

The outcomes for the collected data for both focused questions 1 and 2 are presented in Table 3 .

Outcome of controlled studies answering focused question 1

Linear and volumetric changes in vertical alveolar bone height (mid-buccal)

Parallel studies: Five studies (Fig. 2) reported on changes in the mid-buccal vertical alveolar ridge height dimensions (Iasella et al. 2003; Aimetti et al. 2009; Barone et al. 2013a; Jung et al. 2013; Cardaropoli et al. 2014). There was a moderate level of heterogeneity $\left(I^{2}=55.33 \%, \quad P=0.0839\right)$. The standardised 


\begin{tabular}{|c|c|c|c|c|c|c|c|c|}
\hline References & $\begin{array}{l}\text { Setting (country, } \\
\text { number, type } \\
\text { centre) }\end{array}$ & $\begin{array}{l}\text { Source of } \\
\text { funding, } \\
\text { reported } \\
\text { conflict of } \\
\text { interest }\end{array}$ & Study design & $\begin{array}{l}\text { Who carried } \\
\text { out } \\
\text { procedures }\end{array}$ & $\begin{array}{l}\text { Number } \\
\text { of } \\
\text { patients } \\
\text { (sockets) }\end{array}$ & $\begin{array}{l}\text { Mean age } \pm \\
\text { SD } \\
\text { and/or range }\end{array}$ & $\begin{array}{l}\text { Smokers } \\
\text { included }\end{array}$ & $\begin{array}{l}\text { Socket location and } \\
\text { defect morphology }\end{array}$ \\
\hline $\begin{array}{l}\text { Aimetti et al. } \\
(2009){ }^{\star} \dagger\end{array}$ & Italy, 1, U & Unclear & RCT, Parallel & Unclear & $40(40)$ & $\begin{array}{l}51.27 \pm 8.40 \\
36-68\end{array}$ & $\mathrm{~N}$ & $\begin{array}{l}\text { Anterior maxillary } \\
\text { single-tooth; } 4 \text { walls }\end{array}$ \\
\hline $\begin{array}{l}\text { Barone et al. } \\
(2013 a)^{* \prime} \dagger\end{array}$ & Italy, 1, H & Unclear & RCT, Parallel & Specialists & $58(58)$ & $40.5 ; 20-63$ & $\mathrm{Y}$ & Molar or premolar \\
\hline $\begin{array}{l}\text { Barone et al. } \\
(2013 \mathrm{~b})\end{array}$ & $\begin{array}{l}\text { Italy, Germany, } \\
\text { Spain, 6, U } \\
\text { and P? }\end{array}$ & $\begin{array}{l}\text { Industry, } \\
\text { Unclear }\end{array}$ & $\mathrm{RCT}$, Unclear & Unclear & $38(62)$ & $51 \pm 14$ & $\mathrm{Y}$ & $\begin{array}{l}\text { Molar or premolar; } \\
\text { excluded if facial } \\
\text { soft tissue and } \\
\text { buccal plate } \\
\text { markedly reduced }\end{array}$ \\
\hline $\begin{array}{l}\text { Barone et al. } \\
(2014) \dagger\end{array}$ & Italy, 1, H & $\begin{array}{l}\text { Unclear, } \\
\text { No }\end{array}$ & RCT, Parallel & Specialists & $64(64)$ & $\begin{array}{l}32.7 \pm 12.4 \\
18-47\end{array}$ & Y & $\begin{array}{l}\text { Molar or premolar; } \\
4 \text { walls }\end{array}$ \\
\hline $\begin{array}{l}\text { Beck and } \\
\text { Mealey } \\
\text { (2010) }\end{array}$ & USA, $1, \mathrm{U}$ & $\begin{array}{l}\text { Self- } \\
\text { funded, } \\
\text { Yes }\end{array}$ & CCT, Parallel & Unclear & $33(38)$ & $57.4 ; 39-76$ & $?$ & $\begin{array}{l}\text { Single root; excluded } \\
\text { if }>50 \% \text { of any } \\
\text { socket wall absent }\end{array}$ \\
\hline $\begin{array}{l}\text { Borg and } \\
\text { Mealey } \\
(2015) \dagger\end{array}$ & $\begin{array}{l}\text { USA, Unclear, } \\
\text { Unclear }\end{array}$ & $\begin{array}{l}\text { Industry, } \\
\text { Yes }\end{array}$ & RCT, Parallel & Specialists & $42(42)$ & $52 ; 20-89$ & $\mathrm{~N}$ & $\begin{array}{l}\text { Single-rooted tooth; } \\
\text { excluded if }>50 \% \\
\text { dehiscence }\end{array}$ \\
\hline $\begin{array}{l}\text { Calasans- } \\
\text { Maia et al. } \\
(2014) \dagger\end{array}$ & Brazil, 1, U & Unclear & RCT, Parallel & Unclear & $20(20)$ & $\begin{array}{l}44.55 \pm 10.87 \\
23-60\end{array}$ & $\mathrm{~N}$ & Unclear \\
\hline $\begin{array}{l}\text { Camargo } \\
\text { et al. } \\
(2000)^{*}\end{array}$ & Unclear & $\begin{array}{l}\text { Industry, } \\
\text { Unclear }\end{array}$ & $\begin{array}{l}\mathrm{CCT} \text {, Split- } \\
\text { mouth }\end{array}$ & Unclear & $16(32)$ & $44 \pm 15.9$ & $?$ & Non-molar \\
\hline $\begin{array}{l}\text { Cardaropoli } \\
\text { et al. (2014) }\end{array}$ & Italy, 1, P & Unclear & RCT, Parallel & Unclear & $41(48)$ & $47.2 \pm 12.9$ & Y & $\begin{array}{l}\text { Molar or premolar; } \\
3 \text { intact walls and at } \\
\text { least } 80 \% \text { of fourth } \\
\text { wall intact }\end{array}$ \\
\hline $\begin{array}{l}\text { Cook and } \\
\text { Mealey } \\
\text { (2013) }\end{array}$ & USA, $1, \mathrm{U}$ & $\begin{array}{l}\text { Industry, } \\
\text { No }\end{array}$ & RCT, Parallel & $\begin{array}{c}\text { Specialist } \\
\text { trainees }\end{array}$ & $38(40)$ & $56 ; 23-78$ & Y & $\begin{array}{l}\text { Non-molar; excluded } \\
\text { if bony dehiscence } \\
>50 \% \text { of total } \\
\text { socket depth }\end{array}$ \\
\hline $\begin{array}{l}\text { Coomes et al. } \\
(2014) \dagger\end{array}$ & USA, $1, \mathrm{U}$ & $\begin{array}{l}\text { Industry, } \\
\text { Yes }\end{array}$ & RCT, Parallel & Unclear & $34(34)$ & $19-79$ & Y & $\begin{array}{l}\text { Buccal bone } \\
\text { destruction }\end{array}$ \\
\hline $\begin{array}{l}\text { Crespi et al. } \\
(2009 a) \ddagger\end{array}$ & Italy, 1, H & $\begin{array}{l}\text { Unclear, } \\
\text { No }\end{array}$ & $\begin{array}{l}\text { CCT, Split- } \\
\text { mouth }\end{array}$ & Specialists & $15(45)$ & $51.3 ; 28-72$ & $\mathrm{~N}$ & $\begin{array}{l}\text { Molar or premolar; } 3 \\
\text { bone walls and loss } \\
\text { of buccal plate }\end{array}$ \\
\hline $\begin{array}{l}\text { Crespi et al. } \\
(2011 a) \ddagger\end{array}$ & Italy, 1, H & Unclear & $\begin{array}{l}\text { CCT, Split- } \\
\text { mouth }\end{array}$ & Specialists & $15(45)$ & $53.7 ; 32-70$ & $\mathrm{~N}$ & $\begin{array}{l}\text { One molar or } \\
\text { premolar on each } \\
\text { side of jaw and one } \\
\text { additional randomly } \\
\text { located tooth to be } \\
\text { used as a control }\end{array}$ \\
\hline $\begin{array}{l}\text { Crespi et al. } \\
(2011 b) \ddagger\end{array}$ & Italy, 1, H & Unclear & $\begin{array}{l}\text { RCT, Split- } \\
\text { mouth }\end{array}$ & Specialists & $15(30)$ & $53.7 ; 32-70$ & $\mathrm{~N}$ & $\begin{array}{l}\text { One molar or } \\
\text { premolar on each } \\
\text { side of jaw; } 3 \text { bone } \\
\text { walls and loss of } \\
\text { buccal plate }\end{array}$ \\
\hline $\begin{array}{r}\text { Eskow \& } \\
\text { Mealey } \\
(2014) \ddagger\end{array}$ & USA, $1 ?, \mathrm{U}$ & $\begin{array}{l}\text { Unclear, } \\
\text { No }\end{array}$ & RCT, Parallel & Unclear & $35(35)$ & $54 ; 27-79$ & Y & $\begin{array}{l}\text { Non-molar; excluded } \\
\text { if }>50 \% \text { of socket } \\
\text { wall's vertical } \\
\text { dimension absent }\end{array}$ \\
\hline $\begin{array}{l}\text { Fernandes } \\
\text { et al. (2011) }\end{array}$ & Brazil, 1, U & $\begin{array}{l}\text { Unclear, } \\
\text { No }\end{array}$ & $\begin{array}{l}\text { RCT, Split- } \\
\text { mouth }\end{array}$ & Unclear & $18(36)$ & $33-58$ & $?$ & $\begin{array}{l}\text { Maxillary single- } \\
\text { rooted teeth }\end{array}$ \\
\hline $\begin{array}{l}\text { Festa et al. } \\
(2013)^{*}\end{array}$ & Italy, 1, U & $\begin{array}{l}\text { Unclear, } \\
\text { No }\end{array}$ & $\begin{array}{l}\text { RCT, Split- } \\
\text { mouth }\end{array}$ & Unclear & $15(30)$ & $28-58$ & N & $\begin{array}{l}\text { Premolars; excluded } \\
\text { if buccal or palatal/ } \\
\text { lingual bony wall } \\
\text { fractured/lost }\end{array}$ \\
\hline $\begin{array}{l}\text { Fiorellini } \\
\text { et al. } \\
(2005)^{*} \dagger\end{array}$ & USA?, 8, U & $\begin{array}{l}\text { Industry, } \\
\text { Yes }\end{array}$ & $\mathrm{RCT}$, Parallel & Unclear & $80(95)$ & 47.4 & $?$ & $\begin{array}{l}\text { Non-molar maxillary } \\
\text { teeth; buccal wall } \\
\text { defects }\end{array}$ \\
\hline $\begin{array}{l}\text { Gholami } \\
\text { et al. (2012) }\end{array}$ & Iran, 1?, U? & Unclear & $\begin{array}{l}\text { RCT, Split- } \\
\text { mouth }\end{array}$ & Unclear & $12(28)$ & $\begin{array}{l}44.6 \pm 11.4 \\
21-60\end{array}$ & $?$ & $\begin{array}{l}\text { Non-molar; four-wall } \\
\text { sockets }\end{array}$ \\
\hline $\begin{array}{l}\text { Hoang \& } \\
\text { Mealey } \\
(2012) \dagger\end{array}$ & USA, $1, \mathrm{U}$ & $\begin{array}{l}\text { Self- } \\
\text { funded, } \\
\text { No }\end{array}$ & RCT, Parallel & Unclear & $30(30)$ & $56.1 ; 29-76$ & $?$ & $\begin{array}{l}\text { Molar; excluded if } \\
\text { buccal bony } \\
\text { dehiscence }>50 \% \text { of } \\
\text { length of socket }\end{array}$ \\
\hline
\end{tabular}




\begin{tabular}{|c|c|c|c|c|c|c|}
\hline Materials (details, number of patients/sockets) & $\begin{array}{l}\text { Atraumatic } \\
\text { extraction }\end{array}$ & $\begin{array}{l}\text { Flap } \\
\text { raised }\end{array}$ & $\begin{array}{l}\text { Primary } \\
\text { closure }\end{array}$ & $\begin{array}{l}\text { Pre- or } \\
\text { post- } \\
\text { operative } \\
\text { antibiotics }\end{array}$ & $\begin{array}{l}\text { Healing time } \\
\text { before } \\
\text { measurement/ } \\
\text { biopsy }\end{array}$ & $\begin{array}{l}\text { Dimensions } \\
\text { of ridge } \\
\text { evaluated } \\
\text { by }\end{array}$ \\
\hline Grafting (CS; 22/22) vs. unassisted healing (18/18) & $\mathrm{Y}$ & $\mathrm{N}$ & $\mathrm{N}$ & $\mathrm{Y}$ & 3 & Directly \\
\hline $\begin{array}{l}\text { GBR (corticocancellous porcine bone + collagen barrier; 29/29) vs. } \\
\text { unassisted healing (29/29) }\end{array}$ & $?$ & $\mathrm{~N}$ & $\mathrm{~N}$ & $\mathrm{~N}$ & 4 & Directly \\
\hline $\begin{array}{l}\text { GBR (bovine xenograft + collagen barrier; ?/31; T1) vs. GBR (bovine } \\
\text { xenograft + collagen barrier; ?/31; T2) }\end{array}$ & Y & Y & $\mathrm{N}$ & $?$ & 6 & - \\
\hline $\begin{array}{l}\text { GBR (corticocancellous porcine bone + collagen barrier; 32/32; T1) vs. } \\
\text { GBR (corticocancellous porcine bone + collagen barrier; 32/32; T2) }\end{array}$ & Y & $\begin{array}{l}\text { T1: } N \\
\text { T2: Y }\end{array}$ & $\begin{array}{l}\text { T1: } N \\
\text { T2: Y }\end{array}$ & $\mathrm{Y}$ & 3 & Directly \\
\hline $\begin{array}{l}\text { Grafting (non-freeze-dried cancellous mineralised human bone allograft } \\
+ \text { collagen; } 19 / 22 ; \text { T1) vs. grafting (non-freeze-dried cancellous } \\
\text { mineralised human bone allograft + collagen; } 14 / 16 ; \text { T2) }\end{array}$ & Y & $\mathrm{N}$ & $\mathrm{N}$ & $\mathrm{Y}$ & $\begin{array}{l}\text { Approx } 2.5 \text { or } \\
\text { approx } 5.5\end{array}$ & Directly \\
\hline $\begin{array}{l}\text { GBR (cortical mineralised FDBA + d-PTFE barrier; } 20 / 20 ; \text { T1) vs. GBR } \\
(70 \% \text { cortical mineralised FDBA/30\% cortical DFDBA + d-PTFE barrier; } \\
21 / 21 ; \text { T2) }\end{array}$ & Y & $\mathrm{Y}$ & $\mathrm{N}$ & $\mathrm{Y}$ & $\begin{array}{l}17-21 \text { weeks } \\
\text { (average } \\
19 \text { weeks) }\end{array}$ & Directly \\
\hline Grafting (DBBM; 10/10; T1) vs. grafting (DBBM; 10/10; T2) & Y & $\mathrm{Y}$ & Y & $\mathrm{Y}$ & 6 & Directly \\
\hline Grafting (bioactive glass + CS; $16 / 16)$ vs. unassisted healing $(16 / 16)$ & Y & $\mathrm{Y}$ & $\mathrm{N}$ & $\mathrm{Y}$ & 6 & Directly \\
\hline $\begin{array}{l}\text { GBR (bovine bone mineral blended with collagen + collagen barrier; } \\
21 / 24) \text { vs. unassisted healing (20/24) }\end{array}$ & Y & $\begin{array}{r}\text { T1: Y } \\
\text { C: } N\end{array}$ & $\mathrm{~N}$ & $\mathrm{Y}$ & 4 & Directly \\
\hline $\begin{array}{l}\text { GBR (bovine bone mineral blended with collagen + collagen barrier; } \\
20 / 21 \text {; T1) vs. GBR (hydroxyapatite + collagen barrier; } 18 / 19 ; \mathrm{T} 2 \text { ) }\end{array}$ & Y & $\mathrm{Y}$ & $\mathrm{N}$ & $\mathrm{Y}$ & 4-5 & Directly \\
\hline $\begin{array}{l}\text { Grafting (collagen + rhBMP-2; } 18 / 18 ; \mathrm{T} 1 \text { ) vs. grafting } \\
\text { (collagen; } 16 / 16 ; \mathrm{T} 2 \text { ) }\end{array}$ & Y & $\mathrm{N}$ & $\mathrm{N}$ & $\mathrm{Y}$ & 5 & Both \\
\hline $\begin{array}{l}\text { Grafting (MHA + collagen; } 15 / 15 ; \text { T1) vs. grafting (CS + collagen; } \\
15 / 15 ; \text { T2) vs. unassisted healing (15/15) }\end{array}$ & $?$ & $\mathrm{~N}$ & $\mathrm{~N}$ & $\mathrm{Y}$ & 3 & Other \\
\hline $\begin{array}{l}\text { Grafting (MHA + collagen; } 15 / 15 ; \mathrm{T} 1) \text { vs. grafting (corticocancellous } \\
\text { xenogenic bone + collagen; } 15 / 15 ; \mathrm{T} 2 \text { ) vs. grafting (collagen; } 15 / 15 ; \mathrm{T} 3)\end{array}$ & Y & $\mathrm{N}$ & $\mathrm{N}$ & $Y$ & 4 & - \\
\hline $\begin{array}{l}\text { Grafting (corticocancellous xenogenic bone + collagen; } 15 / 15 \text { ) vs. } \\
\text { unassisted healing }(15 / 15)\end{array}$ & $?$ & $\begin{array}{r}\text { T1: } ? \\
\text { C: } N\end{array}$ & $\begin{array}{l}\text { T1: N } \\
\text { C: ? }\end{array}$ & $Y$ & 4 & - \\
\hline $\begin{array}{l}\text { Grafting (cortical FDBA + collagen; } 17 / 17 ; \mathrm{T} 1 \text { ) vs. grafting (cancellous } \\
\text { FDBA + collagen; } 18 / 18 ; \mathrm{T} 2 \text { ) }\end{array}$ & $Y$ & $\mathrm{~N}$ & $\mathrm{~N}$ & $\mathrm{Y}$ & Approx. 4 & Directly \\
\hline $\begin{array}{l}\text { GBR (anorganic bovine bone matrix with cell-binding peptide P-15 + } \\
\text { acellular dermal matrix barrier; } 18 / 18 ; \mathrm{T} 1 \text { ) vs. GBR (acellular dermal } \\
\text { matrix barrier; } 18 / 18 ; \mathrm{T} \text { ) }\end{array}$ & Y & $Y$ & $\mathrm{~N}$ & $Y$ & 6 & Directly \\
\hline $\begin{array}{l}\text { Grafting (corticocancellous porcine bone + soft cortical membrane; } \\
15 / 15) \text { vs. unassisted healing (15/15) }\end{array}$ & $Y$ & $Y$ & $\begin{array}{r}\text { T1: } N \\
\text { C: } Y\end{array}$ & $Y$ & 6 & Directly \\
\hline $\begin{array}{l}\text { Grafting (collagen sponge with human BMP-2; 22/?; T1) vs. grafting } \\
\text { (collagen sponge with BMP-2; } 21 / \text { ?; T2) vs. grafting (collagen sponge; } \\
\text { 17/?; T3) vs. unassisted healing (20/?) }\end{array}$ & $?$ & $Y$ & Y & $Y$ & 4 & СВCT \\
\hline $\begin{array}{l}\text { GBR (DBBM + collagen barrier; } 12 / 14 ; \text { T1) vs. GBR (nanocrystalline HA } \\
\text { embedded in silica gel matrix + collagen barrier; } 12 / 14 ; \mathrm{T} 2 \text { ) }\end{array}$ & $Y$ & $Y$ & $Y$ & $Y$ & $6-8$ & Directly \\
\hline $\begin{array}{l}\text { Grafting (demineralised bone matrix in a carrier of bovine collagen } \\
\text { and sodium alginate }+ \text { collagen; } 16 / 16 ; \mathrm{T} 1 \text { ) vs. grafting (demineralised } \\
\text { bone matrix in a carrier of bovine collagen and sodium alginate }+ \\
\text { collagen; } 14 / 14 ; \mathrm{T} 2 \text { ) }\end{array}$ & $Y$ & $?$ & $\mathrm{~N}$ & $\mathrm{Y}$ & 4-5 & Directly \\
\hline
\end{tabular}


Table 2. (continued)

\begin{tabular}{|c|c|c|c|c|c|c|c|c|}
\hline References & $\begin{array}{l}\text { Setting (country, } \\
\text { number, type } \\
\text { centre) }\end{array}$ & $\begin{array}{l}\text { Source of } \\
\text { funding, } \\
\text { reported } \\
\text { conflict of } \\
\text { interest }\end{array}$ & Study design & $\begin{array}{l}\text { Who carried } \\
\text { out } \\
\text { procedures }\end{array}$ & $\begin{array}{l}\text { Number } \\
\text { of } \\
\text { patients } \\
\text { (sockets) }\end{array}$ & $\begin{array}{l}\text { Mean age } \pm \\
\text { SD } \\
\text { and/or range }\end{array}$ & $\begin{array}{l}\text { Smokers } \\
\text { included }\end{array}$ & $\begin{array}{l}\text { Socket location and } \\
\text { defect morphology }\end{array}$ \\
\hline $\begin{array}{l}\text { Huh et al. } \\
(2011) \dagger\end{array}$ & $\begin{array}{l}\text { South Korea, 3, } \\
\text { Unclear }\end{array}$ & $\begin{array}{l}\text { Governmental, } \\
\text { Unclear }\end{array}$ & RCT, Parallel & Unclear & 72 (72?) & $\begin{array}{l}52.77 \pm 6.71 \\
35-65\end{array}$ & ? & $\begin{array}{l}\text { Premolar or molar; }<50 \% \\
\text { localised alveolar vertical } \\
\text { bone loss }\end{array}$ \\
\hline $\begin{array}{l}\text { lasella et al. } \\
(2003)^{* \prime \dagger}\end{array}$ & Unclear & Unclear & RCT, Parallel & Unclear & $24(24)$ & $51.5 ; 28-76$ & $?$ & Non-molar \\
\hline $\begin{array}{l}\text { Jung et al. } \\
(2013)^{*}\end{array}$ & $\begin{array}{l}\text { Switzerland, } 2 \\
\text { centres in } 1 \mathrm{U}\end{array}$ & $\begin{array}{l}\text { Institutional } \\
\text { and industry, } \\
\text { No }\end{array}$ & RCT, Parallel & Unclear & $40(40)$ & $\begin{array}{l}\text { Per groups: } \\
48 \pm 15 \\
59 \pm 11 \\
65 \pm 13 \\
49 \pm 14\end{array}$ & Y & $\begin{array}{l}\text { Excluded if }>50 \% \text { buccal } \\
\text { bone height lost }\end{array}$ \\
\hline $\begin{array}{l}\text { Karaca et al. } \\
(2015)^{*}\end{array}$ & Turkey, 2?, U? & Self-funded & $\begin{array}{l}\text { RCT, Split- } \\
\text { mouth }\end{array}$ & Unclear & $10(20)$ & $46.7 ; 36-60$ & ? & Maxillary anterior teeth \\
\hline $\begin{array}{l}\text { Kim et al. } \\
(2014) \dagger\end{array}$ & South Korea, 2, U & $\begin{array}{l}\text { Institutional, } \\
\text { Unclear }\end{array}$ & RCT, Parallel & Unclear & $59(59)$ & $\begin{array}{l}\text { Control } \\
51.18 \pm 10.14 \\
\text { experimental } \\
50.37 \pm 13.45\end{array}$ & $?$ & $\begin{array}{l}\text { Non-molar; }<50 \% \text { bone } \\
\text { loss in all dimensions. }\end{array}$ \\
\hline $\begin{array}{l}\text { Lindhe et al. } \\
(2014) \$\end{array}$ & Unclear & Unclear & $\mathrm{CCT}$, Parallel & Unclear & $24(24)$ & $25-54$ & $?$ & $\begin{array}{l}\text { Excluded if buccal } \\
\text { dehiscence defect } \geq 2 \mathrm{~mm}\end{array}$ \\
\hline $\begin{array}{l}\text { Mardinger } \\
\text { et al. } \\
(2012) \ddagger\end{array}$ & $\begin{array}{l}\text { Israel, Unclear, } \\
U \text { and } P\end{array}$ & Unclear & $\begin{array}{l}\text { Prospective } \\
\text { case series, } \\
\text { Non- } \\
\text { controlled }\end{array}$ & Unclear & $36(43)$ & $50.75 ; 24-75$ & Y & $\begin{array}{l}\text { Site not completely } \\
\text { surrounded by bony } \\
\text { walls; excluded if less } \\
\text { than two bony wall } \\
\text { defects }\end{array}$ \\
\hline $\begin{array}{l}\text { Meloni et al. } \\
\text { (2015); }\end{array}$ & Italy, 2, P & Unclear, No & RCT, Parallel & Specialists & $30(30)$ & $48 ; 26-72$ & $\mathrm{Y}$ & $\begin{array}{l}\text { Maxillary non-molar; } \\
\text { excluded if fenestration } \\
\text { or dehiscence } \geq 3 \mathrm{~mm}\end{array}$ \\
\hline $\begin{array}{l}\text { Neiva et al. } \\
(2008) \dagger\end{array}$ & USA, 1, U & Industry, No & RCT, Parallel & Specialists & $24(24)$ & $25-76$ & $\mathrm{~N}$ & $\begin{array}{l}\text { Maxillary premolars with } \\
>80 \% \text { bone volume in all } \\
\text { dimensions }\end{array}$ \\
\hline Patel $(2013) \dagger$ & $\begin{array}{l}\text { United Kingdom, } \\
1, U\end{array}$ & $\begin{array}{l}\text { Industry, } \\
\text { Unclear }\end{array}$ & RCT, Parallel & Specialists & $26(26)$ & $\begin{array}{l}37.3 \pm 11.4 \\
20-58\end{array}$ & Y & $\begin{array}{l}\text { Non-molar; excluded if } \\
\text { major part of buccal or } \\
\text { palatal wall damaged or } \\
\text { lost }\end{array}$ \\
\hline $\begin{array}{l}\text { Perelman- } \\
\text { Karmon } \\
\text { et al. } \\
(2012) \ddagger\end{array}$ & Unclear & Unclear & RCT, Parallel & Unclear & $23(23)$ & $26-68$ & N & $\begin{array}{l}\text { Non-molar; at least } 50 \% \\
\text { of sockets partially } \\
\text { resorbed/destructed at } \\
\text { one to two walls, but } \\
\text { not circumferentially }\end{array}$ \\
\hline $\begin{array}{l}\text { Pinho et al. } \\
\text { (2006) }\end{array}$ & Brazil, 1, U & Unclear, No & $\begin{array}{l}\text { RCT, Split- } \\
\text { mouth }\end{array}$ & Unclear & $10(20)$ & $46.3 ; 35-60$ & $\mathrm{~N}$ & Maxillary non-molar \\
\hline $\begin{array}{l}\text { Poulias et al. } \\
(2013) \dagger\end{array}$ & USA, $1, U$ & $\begin{array}{l}\text { Self-funded, } \\
\text { No }\end{array}$ & RCT, Parallel & $\begin{array}{c}\text { Specialist } \\
\text { trainees }\end{array}$ & $23(23)$ & $52 \pm 16 ; 26-77$ & $\mathrm{Y}$ & Non-molar \\
\hline $\begin{array}{l}\text { Serino et al. } \\
(2003) \ddagger\end{array}$ & Unclear & Unclear & CCT, Unclear & Unclear & $36(39)$ & $35-64$ & $?$ & Unclear \\
\hline $\begin{array}{l}\text { Vance et al. } \\
(2004) \dagger\end{array}$ & Unclear & $\begin{array}{l}\text { Industry, } \\
\text { Unclear }\end{array}$ & RCT, Parallel & Unclear & $24(24)$ & 56 & $?$ & Non-molar \\
\hline $\begin{array}{l}\text { Wallace et al. } \\
(2013) \$\end{array}$ & Unclear & Industry, Yes & CCT, Parallel & Unclear & $30(34)$ & $18-70$ & N & $\begin{array}{l}18 \text { intact and } 16 \text { sockets } \\
\text { with buccal wall defects }\end{array}$ \\
\hline $\begin{array}{l}\text { Wood and } \\
\text { Mealey } \\
(2012) \dagger\end{array}$ & USA, $1, \mathrm{U}$ & Industry, No & RCT, Parallel & $\begin{array}{r}\text { Specialist } \\
\text { trainees }\end{array}$ & $33(33)$ & $56.7 ; 20-78$ & $\mathrm{~N}$ & Single-rooted non-molar \\
\hline
\end{tabular}

$\mathrm{U}$, university; $\mathrm{H}$, hospital, P, private practice; $\mathrm{Y}$, yes; N, no; ?, unclear; RCT, randomised clinical trial; CCT, controlled clinical trial; GBR, guided bone regeneration; $\mathrm{T} 1$, test group $1 ; \mathrm{T} 2$, test group $2 ; \mathrm{T} 3$, test group 3 ; $\mathrm{C}$, control group; $\mathrm{MHA}$, magnesium-enriched hydroxyapatite; CS, calcium sulphate; FDBA, freeze-dried bone allograft; DBBM, deproteinised bovine bone mineral; HA, hydroxyapatite; DFDBA, demineralised freeze-dried bone allograft; d-PTFE, dense polytetrafluoroethylene; $\beta$-TCP, Beta-tricalcium phosphate.

All studies included for question 2; highlighted studies included for question 1.

*Selected for meta-analysis question 1.

$\dagger$ Selected for meta-analysis question 2.

$\$$ Included only for histological data. 
Grafting ( $\beta$-TCP/HA + ErhBMP-2; 36/?; T1) vs. grafting ( $\beta$-TCP/HA; 36/?; T2)

GBR (FDBA + collagen barrier; 12/12) vs. unassisted healing (12/12)

Grafting ( $\beta$-TCP particles with poly(lactide co-glycolide) coating;

10/10; T1) vs. sealing (DBBM with $10 \%$ collagen + porcine collagen

matrix (Mucograft); 10/10; T2) vs. sealing (DBBM with $10 \%$ collagen +

autogenous soft tissue graft; 10/10; T3) vs. unassisted healing (10/10)

Sealing (free gingival graft from palate; 10/10) vs. unassisted healing (10/10)

GBR (demineralised human bone matrix + rhBMP-2 + collagen barrier;

29/29; T1) vs. GBR (demineralised human bone matrix + rhBMP-2 +

collagen barrier; 30/30; T2)

Sealing (DBBM + Mucograft; 13/13; T1) vs. sealing (Mucograft; 11/11; T2)

Sealing (porous bovine xenograft + intrasocket reactive soft tissue; $36 / 43)$

\begin{tabular}{|c|c|c|c|c|c|}
\hline $\begin{array}{l}\text { Atraumatic } \\
\text { extraction }\end{array}$ & $\begin{array}{l}\text { Flap } \\
\text { raised }\end{array}$ & $\begin{array}{l}\text { Primary } \\
\text { closure }\end{array}$ & $\begin{array}{l}\text { Pre- or } \\
\text { post- } \\
\text { operative } \\
\text { antibiotics }\end{array}$ & $\begin{array}{l}\text { Healing time } \\
\text { before } \\
\text { measurement/ } \\
\text { biopsy }\end{array}$ & $\begin{array}{l}\text { Dimensior } \\
\text { of ridge } \\
\text { evaluated } \\
\text { by }\end{array}$ \\
\hline$?$ & $?$ & $?$ & $?$ & 3 & $\mathrm{CBCT}$ \\
\hline Y & $Y$ & $\mathrm{~N}$ & $Y$ & 4 or 6 & Directly \\
\hline Y & $?$ & $\begin{array}{l}\text { T1; N } \\
\text { T2: Y } \\
\text { T3: Y } \\
\text { C: N }\end{array}$ & $Y$ & 6 & $\mathrm{CBCT}$ \\
\hline Y & $\mathrm{N}$ & $\begin{array}{l}\text { T: Y } \\
\text { C: N }\end{array}$ & $Y$ & 3 & $\mathrm{CBCT}$ \\
\hline Y & $Y$ & $?$ & $Y$ & 3 & $\mathrm{CBCT}$ \\
\hline Y & $\mathrm{N}$ & $Y$ & $?$ & 6 & - \\
\hline Y & $\mathrm{N}$ & $\mathrm{Y}$ & $Y$ & 6 & - \\
\hline
\end{tabular}

Sealing (DBBM + epithelial connective tissue graft from palate; 15/15;

T1) vs. sealing (DBBM + porcine collagen matrix; 15/15; T2)

Grafting (anorganic bovine-derived HA matrix combined with a synthetic cell-binding peptide P-15 + collagen; $12 / 12 ; \mathrm{T} 1$ ) vs. grafting (collagen; $12 / 12 ; \mathrm{T} 2)$

GBR ( $60 \%$ HA + 40\% b-tricalcium phosphate + collagen barrier; 13/13;

T1) vs. GBR (DBBM + collagen barrier; 13/13; T2)

GBR (bovine bone mineral + collagen barrier; 11/11; T1) vs. grafting

(bovine mineral bone; 12/12; T2)

GBR (autograft + titanium barrier; 10/10; T1) vs. GBR (titanium barrier; 10/10; T2)

GBR (cancellous allograft + resorbable polylactide barrier; 12/12; T1) vs.

GBR (cancellous allograft + bovine xenograft + resorbable polylactide

barrier; 11/11; T2)

Grafting (polylactide-polyglycolide acid sponge; 24/26) vs. unassisted

healing (12/13)

Grafting (carboxymethylcellulose + CS + DFDBA; 12/12; T1) vs. GBR

(bovine bone mineral + collagen barrier; 12/12; T2)

GBR (allograft + rhPDGF-BB + resorbable acellular dermal matrix barrier;

?/19; T1) vs. GBR (allograft + saline + acellular dermal matrix barrier;

?/15; T2)

Grafting (DFDBA + collagen; 17/17; T1) vs. grafting (FDBA + collagen; 16/16; T2)

$N$

N

Y

N

$?$

N

Y
Y

T1: N

T2: ?

N

Y

?

Y
N

$\mathrm{N}$

Y

N

Y

Y

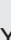

5

3.7

8

Directly

\section{CBCT}

Directly

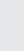

\section{6}

Directly

Directly

Directly

Directly

4

$\begin{array}{lll}4 & 4.7\end{array}$

Directly 
Table 3. Study outcomes of included papers (I)

\begin{tabular}{|c|c|c|c|c|c|c|}
\hline References & Comparison & $\begin{array}{l}\text { Changes in vertical } \\
\text { alveolar ridge } \\
\text { dimensions - } \\
\text { mid-buccal }\end{array}$ & $\begin{array}{l}\text { Changes in vertical } \\
\text { alveolar ridge } \\
\text { dimensions - } \\
\text { proximal }\end{array}$ & $\begin{array}{l}\text { Changes in } \\
\text { horizontal alveolar } \\
\text { ridge dimensions }\end{array}$ & Histology (\%) & $\begin{array}{l}\text { Changes in } \\
\text { keratinised tissues } \\
\text { dimensions }\end{array}$ \\
\hline $\begin{array}{l}\text { Aimetti } \\
\text { et al. } \\
(2009)^{*} \uparrow\end{array}$ & $\begin{array}{l}\text { Grafting vs. } \\
\text { unassisted } \\
\text { healing }\end{array}$ & $\begin{array}{c}\mathrm{T}-0.5 \pm 1.1 \\
\mathrm{C}-1.2 \pm 0.6\end{array}$ & $\begin{array}{c}\mathrm{T}-0.2 \pm 0.6 \\
\mathrm{C}-0.5 \pm 0.9\end{array}$ & $\begin{array}{l}\mathrm{T}-2 \pm 1.1 \\
\mathrm{C}-3.2 \pm 1.8\end{array}$ & $\begin{array}{l}\text { T } 58.8 \pm 3.5 \text { trabecular bone } \\
\text { area fraction } \\
\text { C } 47.2 \pm 7.7 \text { trabecular bone } \\
\text { area fraction }\end{array}$ & - \\
\hline $\begin{array}{l}\text { Barone } \\
\text { et al. } \\
(2013 a)^{* / \dagger} \dagger\end{array}$ & $\begin{array}{l}\text { GBR vs. } \\
\text { unassisted } \\
\text { healing }\end{array}$ & $\begin{array}{r}\mathrm{T}-1.1 \pm 0.96 \\
\mathrm{C}-2.1 \pm 0.6\end{array}$ & $\begin{array}{c}\mathrm{T}-0.3 \pm 0.76 \\
\mathrm{C}-1 \pm 0.7\end{array}$ & $\begin{array}{c}\mathrm{T}-1.6 \pm 0.55 \\
\mathrm{C}-3.6 \pm 0.72\end{array}$ & - & $\begin{array}{l}\text { Changes in width of } \\
\text { keratinised gingiva } \\
T+1.14 \pm 0.8 \\
\mathrm{C}+0.73 \pm 0.8\end{array}$ \\
\hline $\begin{array}{l}\text { Barone } \\
\text { et al. } \\
(2013 b)\end{array}$ & $\begin{array}{l}\text { GBR (T1) vs. } \\
\text { GBR (T2) }\end{array}$ & - & - & - & $\begin{array}{l}\text { T1 } 28.5 \pm 20 \mathrm{VB} \\
\text { T2 } 31.4 \pm 18.1 \mathrm{VB}\end{array}$ & - \\
\hline $\begin{array}{l}\text { Barone } \\
\text { et al. } \\
(2014) \dagger\end{array}$ & $\begin{array}{l}\text { GBR (T1) vs. } \\
\text { GBR (T2) }\end{array}$ & $\begin{array}{l}\mathrm{T} 1-1.1 \pm 0.9 \\
\mathrm{~T} 2-0.6 \pm 0.7\end{array}$ & $\begin{array}{l}\mathrm{T} 1-0.3 \pm 0.7 \\
\mathrm{~T} 2-0.4 \pm 0.5\end{array}$ & $\begin{array}{l}\mathrm{T} 1-1.7 \pm 0.6 \\
\mathrm{~T} 2-3.5 \pm 0.9\end{array}$ & - & $\begin{array}{l}\text { Changes in width of } \\
\text { keratinised gingiva } \\
\mathrm{T} 1+1.8 \pm 0.8 \\
\mathrm{~T} 2-1.7 \pm 0.6\end{array}$ \\
\hline $\begin{array}{l}\text { Beck and } \\
\text { Mealey } \\
\text { (2010) }\end{array}$ & $\begin{array}{l}\text { Grafting (T1) } \\
\text { vs. grafting } \\
\text { (T2) }\end{array}$ & $\begin{array}{l}\text { Data per socket } \\
\text { T1 } 0.32 \pm 2.61 \\
\text { T2-0.37 } \pm 1.46\end{array}$ & - & $\begin{array}{l}\text { Data per socket } \\
\text { T1 }-1.43 \pm 1.89 \\
\mathrm{~T} 2-1.47 \pm 1.89\end{array}$ & $\begin{array}{l}\mathrm{T} 145 \pm 19.8 \% \text { new VB; } \\
41.3 \pm 14.6 \% \mathrm{CT} ; \\
13.5 \pm 12.2 \% \text { RGM } \\
\text { T2 } 45.8 \pm 22.4 \% \text { new VB; } \\
39.6 \pm 13.0 \% \mathrm{CT} ; \\
14.6 \pm 12.9 \% \text { RGM }\end{array}$ & - \\
\hline $\begin{array}{l}\text { Borg and } \\
\text { Mealey } \\
(2015) \dagger\end{array}$ & $\begin{array}{l}\text { GBR (T1) vs. } \\
\text { GBR (T2) }\end{array}$ & $\begin{array}{c}\mathrm{T} 1-0.25 \pm 1.85 \\
\mathrm{~T} 20.26 \pm 2.08\end{array}$ & - & $\begin{array}{l}\mathrm{T} 1-1.63 \pm 1.18 \\
\mathrm{~T} 2-1.19 \pm 1.36\end{array}$ & $\begin{array}{l}\text { T1 } 24.69 \pm 15.92 \mathrm{VB} ; \\
27.04 \pm 13.62 \mathrm{RGM} ; \\
48.27 \pm 14.16 \mathrm{CT} / \text { other } \\
\text { T2 } 36.16 \pm 11.91 \mathrm{VB} ; \\
18.24 \pm 12.47 \mathrm{RGM} ; \\
45.38 \pm 11.09 \mathrm{CT} / \text { other }\end{array}$ & - \\
\hline $\begin{array}{l}\text { Calasans- } \\
\text { Maia } \\
\text { et al. } \\
(2014) \dagger\end{array}$ & $\begin{array}{l}\text { Grafting (T1) } \\
\text { vs. grafting } \\
\text { (T2) }\end{array}$ & - & - & $\begin{array}{l}\mathrm{T} 1-0.29 \pm 0.14 \\
\mathrm{~T} 2-0.39 \pm 0.14\end{array}$ & $\begin{array}{l}\text { T1 } 33.6 \pm 7.1 \text { new VB area } \\
\text { fraction; } 32.3 \pm 8.8 \mathrm{CT} ; \\
10.6 \pm 16.2 \mathrm{RGM} \\
\text { T2 } 19.3 \pm 22.5 \text { new VB area } \\
\text { fraction; } 49.9 \pm 14 \mathrm{CT} ; \\
22.5 \pm 7.9 \mathrm{RGM}\end{array}$ & - \\
\hline $\begin{array}{l}\text { Camargo } \\
\text { et al. } \\
(2000)^{*}\end{array}$ & $\begin{array}{l}\text { Grafting vs. } \\
\text { unassisted } \\
\text { healing }\end{array}$ & $\begin{array}{c}\mathrm{T}-0.38 \pm 3.18 \\
\mathrm{C}-1 \pm 2.25\end{array}$ & - & $\begin{array}{c}T-3.48 \pm 2.68 \\
C-3.06 \pm 2.41\end{array}$ & - & - \\
\hline $\begin{array}{l}\text { Cardaropoli } \\
\text { et al. } \\
\text { (2014) }\end{array}$ & $\begin{array}{l}\text { GBR vs. } \\
\text { unassisted } \\
\text { healing }\end{array}$ & $\begin{array}{r}\text { Data per socket } \\
\mathrm{T}-0.56 \pm 0.45 \\
\mathrm{C}-1.67 \pm 0.43\end{array}$ & - & $\begin{array}{l}\text { Data per socket } \\
\mathrm{T}-0.71 \pm 0.91 \\
\mathrm{C}-4.04 \pm 0.69\end{array}$ & - & - \\
\hline $\begin{array}{l}\text { Cook and } \\
\text { Mealey } \\
(2013)\end{array}$ & $\begin{array}{l}\text { GBR (T1) vs. } \\
\text { GBR (T2) }\end{array}$ & $\begin{array}{l}\text { Data per socket } \\
\text { T1 }-0.14 \pm 2.21 \\
\text { T2 } 0.03 \pm 2.81\end{array}$ & - & $\begin{array}{l}\text { Data per socket } \\
\text { T1 }-1.57 \pm 1.21 \\
\text { T2 }-1.16 \pm 1.44\end{array}$ & $\begin{array}{l}\text { T1 } 32.8 \pm 14.7 \text { bone; } \\
13.4 \pm 11.6 \text { RGM; } 53.7 \pm 6.8 \\
\text { CT/other } \\
\text { T2 } 47 \pm 9.1 \text { bone; RGM not } \\
\text { detected; } 53 \pm 9.1 \text { CT/other }\end{array}$ & - \\
\hline $\begin{array}{l}\text { Coomes } \\
\text { et al. } \\
(2014) \dagger\end{array}$ & $\begin{array}{l}\text { Grafting (T1) } \\
\text { vs. grafting } \\
\text { (T2) }\end{array}$ & $\begin{array}{l}\text { T1 } 4.75 \pm 2.65 \\
\text { T2 } 1.85 \pm 3.58\end{array}$ & - & $\begin{array}{r}\mathrm{T} 1-2.07 \pm 1.17 \\
\mathrm{~T} 2-3.4 \pm 1.73\end{array}$ & - & - \\
\hline $\begin{array}{l}\text { Crespi } \\
\text { et al. } \\
(2009 a) \ddagger\end{array}$ & $\begin{array}{l}\text { Grafting (T1) } \\
\text { vs. grafting } \\
\text { (T2) vs. } \\
\text { unassisted } \\
\text { healing }\end{array}$ & - & - & - & $\begin{array}{l}\mathrm{T} 140 \pm 2.7 \mathrm{VB} ; 41.3 \pm 1.3 \mathrm{CT} ; \\
20.2 \pm 3.2 \mathrm{RGM} \\
\mathrm{T} 245 \pm 6.5 \mathrm{VB} ; 41.5 \pm 6.7 \\
\mathrm{CT} ; 13.9 \pm 3.4 \mathrm{RGM} \\
\mathrm{C} 32.8 \pm 5.8 \mathrm{VB} ; 64.6 \pm 6.8 \\
\mathrm{CT}\end{array}$ & - \\
\hline $\begin{array}{l}\text { Crespi } \\
\text { et al. } \\
(2011 a) \ddagger\end{array}$ & $\begin{array}{l}\text { Grafting (T1) } \\
\text { vs. grafting } \\
\text { (T2) vs. } \\
\text { grafting (T3) }\end{array}$ & - & - & - & $\begin{array}{l}\mathrm{T} 136.5 \pm 2.6 \mathrm{VB} ; 33.3 \pm 1.5 \\
\mathrm{CT} ; 32.2 \pm 3.2 \mathrm{RGM} \\
\mathrm{T} 238.0 \pm 16.2 \mathrm{VB} ; 25.3 \pm 9.4 \\
\mathrm{CT} ; 36.6 \pm 4.8 \mathrm{RGM} \\
\text { T3 } 30.3 \pm 4.8 \mathrm{VB} ; 58.3 \pm 7.1 \\
\mathrm{CT}\end{array}$ & - \\
\hline $\begin{array}{l}\text { Crespi } \\
\text { et al. } \\
(2011 b)\end{array}$ & $\begin{array}{l}\text { Grafting vs. } \\
\text { unassisted } \\
\text { healing }\end{array}$ & - & - & - & $\begin{array}{l}\text { T } 39.6 \pm 9.4 \mathrm{VB} ; 26.0 \pm 9.9 \mathrm{CT} \\
34.4 \pm 5.1 \mathrm{RGM} \\
\text { C } 29.5 \pm 5 \mathrm{VB} ; 57.7 \pm 6.9 \mathrm{CT}\end{array}$ & - \\
\hline $\begin{array}{l}\text { Eskow \& } \\
\text { Mealey } \\
(2014) \$\end{array}$ & $\begin{array}{l}\text { Grafting (T1) } \\
\text { vs. grafting } \\
\text { (T2) }\end{array}$ & - & - & - & $\begin{array}{l}\text { Mean (range) } \\
\text { T1 } 16.1 \text { (12.1-30.3) new } \\
\text { bone; } 28.4(18.5-37.5) \text { RGM; } \\
52.9 \text { (47.4-57.1) CT/other } \\
\text { T2 } 13(10.1-31) \text { new bone; } \\
19.9 \text { (15.8-24.3) RGM; } \\
62.8 \text { (50.9-68.5) CT/other }\end{array}$ & - \\
\hline
\end{tabular}




\begin{tabular}{|c|c|c|c|c|c|c|}
\hline References & Comparison & $\begin{array}{l}\text { Changes in vertical } \\
\text { alveolar ridge } \\
\text { dimensions - } \\
\text { mid-buccal }\end{array}$ & $\begin{array}{l}\text { Changes in vertical } \\
\text { alveolar ridge } \\
\text { dimensions - } \\
\text { proximal }\end{array}$ & $\begin{array}{l}\text { Changes in } \\
\text { horizontal alveolar } \\
\text { ridge dimensions }\end{array}$ & Histology (\%) & $\begin{array}{l}\text { Changes in } \\
\text { keratinised tissues } \\
\text { dimensions }\end{array}$ \\
\hline $\begin{array}{l}\text { Fernandes } \\
\text { et al. } \\
\text { (2011) }\end{array}$ & $\begin{array}{l}\text { GBR (T1) vs. } \\
\text { GBR (T2) }\end{array}$ & $\begin{array}{l}\mathrm{T} 1-1.2 \pm 2 \\
\mathrm{~T} 2-1.5 \pm 1.2\end{array}$ & - & $\begin{array}{r}\mathrm{T} 1-2.5 \pm 1.8 \\
\mathrm{~T} 2-3.4 \pm 1.4\end{array}$ & - & - \\
\hline $\begin{array}{l}\text { Festa et al. } \\
(2013)^{*}\end{array}$ & $\begin{array}{l}\text { Grafting vs. } \\
\text { unassisted } \\
\text { healing }\end{array}$ & $\begin{array}{c}\mathrm{T}-0.6 \pm 1.4 \\
\mathrm{C}-3.1 \pm 1.3\end{array}$ & $\begin{array}{l}\mathrm{T}-0.3 \pm 0.8 \\
\mathrm{C}-0.4 \pm 1.2\end{array}$ & $\begin{array}{c}\mathrm{T}-1.8 \pm 1.3 \\
\mathrm{C}-3.7 \pm 1.2\end{array}$ & - & $\begin{array}{l}\text { No changes to } \\
\text { keratinised } \\
\text { margin }\end{array}$ \\
\hline $\begin{array}{l}\text { Fiorellini } \\
\text { et al. } \\
(2005)^{* \bullet \dagger}\end{array}$ & $\begin{array}{l}\text { Grafting (T1) } \\
\text { vs. grafting } \\
\text { (T2) vs. } \\
\text { grafting (T3) } \\
\text { vs. unassisted } \\
\text { healing }\end{array}$ & $\begin{array}{c}\mathrm{T} 1-0.6 \pm 1.4 \\
\mathrm{~T} 2-0 \pm 1.2 \\
\mathrm{~T} 3-1 \pm 1.4 \\
\mathrm{C}-1.2 \pm 1.2\end{array}$ & - & - & $\begin{array}{l}\text { Only descriptive } \\
\text { Bone structure of } \\
\text { approximately two-thirds of } \\
\text { samples was exclusively } \\
\text { trabecular. Remodelling of } \\
\text { woven bone into lamellar } \\
\text { bone was the most common } \\
\text { observation }\end{array}$ & - \\
\hline $\begin{array}{l}\text { Gholami } \\
\text { et al. } \\
\text { (2012) }\end{array}$ & $\begin{array}{l}\text { GBR (T1) vs. } \\
\text { GBR (T2) }\end{array}$ & - & - & $\begin{array}{l}\text { Data per socket } \\
\text { T1 }-1.1 \pm 1 \\
\text { T2 }-0.9 \pm 0.6\end{array}$ & $\begin{array}{l}\text { T1 } 27.4 \pm 12.4 \text { total bone; } \\
20.6 \pm 9.9 \text { RGM } \\
\text { T2 } 28.6 \pm 12.5 \text { total bone; } \\
13.7 \pm 8.1 \text { RGM }\end{array}$ & - \\
\hline $\begin{array}{l}\text { Hoang \& } \\
\text { Mealey } \\
(2012) \dagger\end{array}$ & $\begin{array}{l}\text { Grafting (T1) } \\
\text { vs. grafting } \\
\text { (T2) }\end{array}$ & $\begin{array}{l}\mathrm{T} 1-0.1 \pm 1.8 \\
\mathrm{~T} 20 \pm 1.9\end{array}$ & - & $\begin{array}{l}\mathrm{T} 1-1.4 \pm 1.5 \\
\mathrm{~T} 2-1.3 \pm 1.5\end{array}$ & $\begin{array}{l}\text { T1 } 48.8 \text { VB; } 8.2 \text { RGM; } 43.1 \mathrm{CT} \\
\text { T2 } 52.7 \text { VB; 5.4 RGM; } 41.9 \text { CT }\end{array}$ & - \\
\hline $\begin{array}{l}\text { Huh et al. } \\
(2011) \dagger\end{array}$ & $\begin{array}{l}\text { Grafting (T1) } \\
\text { vs. grafting } \\
\text { (T2) }\end{array}$ & $\begin{array}{l}\mathrm{T} 1-0.1 \pm 1 \\
\mathrm{~T} 2-1.1 \pm 1.4\end{array}$ & - & - & - & - \\
\hline $\begin{array}{l}\text { lasella } \\
\text { et al. } \\
(2003)^{*+\dagger} \dagger\end{array}$ & $\begin{array}{l}\text { GBR vs. } \\
\text { unassisted } \\
\text { healing }\end{array}$ & $\begin{array}{l}\text { T } 1.3 \pm 2 \\
\mathrm{C}-0.9 \pm 1.6\end{array}$ & $\begin{array}{c}\mathrm{T}-0.1 \pm 0.7 \\
\mathrm{C}-1 \pm 0.8\end{array}$ & $\begin{array}{l}T-1.2 \pm 0.9 \\
C-2.6 \pm 2.3\end{array}$ & $\begin{array}{l}\text { T } 28 \pm 14 \text { VB; } 37 \pm 18 \text { non- } \\
\text { vital; } 26 \pm 11 \text { trabecular; } \\
9 \pm 6 \text { amorphous } \\
\text { C } 54 \pm 12 \text { VB; - non-vital; } \\
34 \pm 12 \text { trabecular; } 12 \pm 9 \\
\text { amorphous }\end{array}$ & $\begin{array}{l}\text { Changes in buccal } \\
\text { gingival thickness } \\
\mathrm{T}-0.1 \pm 0.5 \\
\mathrm{C}+0.4 \pm 0.6\end{array}$ \\
\hline $\begin{array}{l}\text { Jung et al. } \\
(2013)^{*}\end{array}$ & $\begin{array}{l}\text { Grafting (T1) } \\
\text { vs. sealing } \\
\text { (T2) vs. } \\
\text { sealing (T3) } \\
\text { vs. unassisted } \\
\text { healing }\end{array}$ & $\begin{array}{l}\mathrm{T} 1-2 \pm 2.4 \\
\mathrm{~T} 20 \pm 1.2 \\
\mathrm{~T} 31.2 \pm 2.9 \\
\mathrm{C}-0.5 \pm 0.9\end{array}$ & - & $\begin{array}{l}\mathrm{T} 1-6.1 \pm 2.5 \\
\mathrm{~T} 2-1.2 \pm 0.8 \\
\mathrm{~T} 3-1.4 \pm 1 \\
\mathrm{C}-3.3 \pm 2\end{array}$ & - & - \\
\hline $\begin{array}{l}\text { Karaca } \\
\text { et al. } \\
(2015)\end{array}$ & $\begin{array}{l}\text { Sealing vs. } \\
\text { unassisted } \\
\text { healing }\end{array}$ & $\begin{aligned} \mathrm{T}-0.012 & \pm 1.24 \\
\mathrm{C}-1.42 & \pm 1.5\end{aligned}$ & - & - & - & - \\
\hline $\begin{array}{l}\text { Kim } \\
\text { et al. } \\
(2014) \dagger\end{array}$ & $\begin{array}{l}\text { GBR (T1) vs. } \\
\text { GBR (T2) }\end{array}$ & $\begin{array}{l}\mathrm{T} 1-1.2 \pm 0.8 \\
\mathrm{~T} 2-1.5 \pm 1.1\end{array}$ & - & $\begin{array}{l}\mathrm{T} 1-1.1 \pm 1.3 \\
\mathrm{~T} 2-1.2 \pm 1.3\end{array}$ & - & - \\
\hline $\begin{array}{l}\text { Lindhe } \\
\text { et al. } \\
\text { (2014) }\end{array}$ & $\begin{array}{l}\text { Sealing (T1) } \\
\text { vs. sealing } \\
\text { (T2) }\end{array}$ & - & - & - & $\begin{array}{l}\text { T1 } 39.9 \pm 8.6 \text { mineralised } \\
\text { bone; } 1.8 \pm 2.5 \text { bone } \\
\text { marrow; } 1.6 \pm 1.8 \text { osteoid; } \\
32.4 \pm 9.2 \text { fibrous tissue } \\
\text { T2 } 57.4 \pm 12.4 \text { mineralised } \\
\text { bone; } 7.1 \pm 6.1 \text { bone } \\
\text { marrow; } 7.3 \pm 4.9 \text { osteoid; } \\
23.1 \pm 16.3 \text { fibrous tissue; } \\
3.3 \pm 1.7 \text { vascular tissue }\end{array}$ & - \\
\hline $\begin{array}{l}\text { Mardinger } \\
\text { et al. } \\
(2012) \ddagger\end{array}$ & Sealing & - & - & - & $\begin{array}{c}40 \pm 19 \text { bone (13.7-74.8); } \\
25.7 \pm 13(0.6-51) \mathrm{RGM} ; \\
34.3 \pm 15(13.8-71.9) \mathrm{CT}\end{array}$ & - \\
\hline $\begin{array}{l}\text { Meloni } \\
\text { et al. } \\
\text { (2015) }\end{array}$ & $\begin{array}{l}\text { Sealing (T1) } \\
\text { vs. Sealing } \\
\text { (T2) }\end{array}$ & $\begin{array}{l}\mathrm{T} 1-1.6 \pm 0.7 \\
\mathrm{~T} 2-1.5 \pm 0.6\end{array}$ & - & $\begin{array}{c}\mathrm{T} 1-0.5 \pm 0.3 \\
\mathrm{~T} 2-0.7 \pm 0.3\end{array}$ & - & - \\
\hline $\begin{array}{l}\text { Neiva } \\
\text { et al. } \\
(2008) \dagger\end{array}$ & $\begin{array}{l}\text { Grafting (T1) } \\
\text { vs. grafting } \\
\text { (T2) }\end{array}$ & $\begin{array}{c}\mathrm{T} 10.2 \pm 1.8 \\
\mathrm{~T} 2-0.6 \pm 1\end{array}$ & - & $\begin{array}{l}\mathrm{T} 1-1.3 \pm 1 \\
\mathrm{~T} 2-1.4 \pm 1.1\end{array}$ & $\begin{array}{l}\text { T1 } 29.9 \pm 8.5 \mathrm{VB} ; 65.3 \pm 6.4 \\
\text { bone marrow and fibrous } \\
\text { tissue; } 6.3 \mathrm{RGM} \\
\mathrm{T} 236.5 \pm 7.7 \mathrm{VB} ; 62.7 \pm 7.4 \\
\text { bone marrow and fibrous } \\
\text { tissue }\end{array}$ & - \\
\hline $\begin{array}{l}\text { Patel } \\
(2013) \dagger\end{array}$ & $\begin{array}{l}\text { GBR (T1) vs. } \\
\text { GBR (T2) }\end{array}$ & - & $\begin{array}{l}\mathrm{T} 1-0.4 \pm 1 \\
\mathrm{~T} 20.2 \pm 0.7\end{array}$ & $\begin{array}{l}\mathrm{T} 1-1.1 \pm 1 \\
\mathrm{~T} 2-2.1 \pm 1\end{array}$ & $\begin{array}{l}\text { Only descriptive } \\
\text { Similar characteristics both } \\
\text { groups. Newly formed bone } \\
\text { mainly at apical part of } \\
\text { biopsy. In coronal part, } \\
\text { particles surrounded by }\end{array}$ & - \\
\hline
\end{tabular}


Table 3. (continued)

\begin{tabular}{|c|c|c|c|c|c|c|}
\hline References & Comparison & $\begin{array}{l}\text { Changes in vertical } \\
\text { alveolar ridge } \\
\text { dimensions - } \\
\text { mid-buccal }\end{array}$ & $\begin{array}{l}\text { Changes in vertical } \\
\text { alveolar ridge } \\
\text { dimensions - } \\
\text { proximal }\end{array}$ & $\begin{array}{l}\text { Changes in } \\
\text { horizontal alveolar } \\
\text { ridge dimensions }\end{array}$ & Histology (\%) & $\begin{array}{l}\text { Changes in } \\
\text { keratinised tissues } \\
\text { dimensions }\end{array}$ \\
\hline & & & & & $\begin{array}{l}\text { dense connective tissue with } \\
\text { no signs of inflammation. No } \\
\text { active resorption of graft } \\
\text { particles }\end{array}$ & - \\
\hline $\begin{array}{l}\text { Perelman- } \\
\text { Karmon } \\
\text { et al. } \\
(2012) \ddagger\end{array}$ & $\begin{array}{l}\text { GBR (T1) vs. } \\
\text { grafting (T2) }\end{array}$ & - & - & - & $\begin{array}{l}\text { T1 } 40.8 \pm 10.6 \text { total bone } \\
\text { fraction } \\
\text { T2 } 29.7 \pm 7.2 \text { total bone } \\
\text { fraction }\end{array}$ & - \\
\hline $\begin{array}{l}\text { Pinho et al. } \\
(2006)\end{array}$ & $\begin{array}{l}\text { GBR (T1) vs. } \\
\text { GBR (T2) }\end{array}$ & - & - & $\begin{array}{c}\mathrm{T} 1-1.4 \pm 1 \\
\mathrm{~T} 2-1.4 \pm 2\end{array}$ & - & - \\
\hline $\begin{array}{l}\text { Poulias } \\
\text { et al. } \\
(2013) \dagger\end{array}$ & $\begin{array}{l}\text { GBR (T1) vs. } \\
\text { GBR (T2) }\end{array}$ & $\begin{array}{c}\text { T1 } 0.5 \pm 2.9 \\
\text { T2 } 0.3 \pm 2.6\end{array}$ & $\begin{array}{c}\mathrm{T} 1-0.5 \pm 0.4 \\
\mathrm{~T} 2-0.6 \pm 0.4\end{array}$ & $\begin{array}{c}\mathrm{T} 1-1.6 \pm 0.8 \\
\mathrm{~T} 2-0.3 \pm 0.9\end{array}$ & $\begin{array}{l}\mathrm{T} 135 \pm 16 \mathrm{VB} ; 21 \pm 13 \text { non- } \\
\text { vital bone; } 44 \pm 9 \text { trabecular } \\
\text { space } \\
\mathrm{T} 240 \pm 16 \mathrm{VB} ; 17 \pm 11 \text { non- } \\
\text { vital bone; } 43 \pm 12 \\
\text { trabecular space }\end{array}$ & - \\
\hline $\begin{array}{l}\text { Serino } \\
\text { et al. } \\
(2003) \ddagger\end{array}$ & $\begin{array}{l}\text { Grafting vs. } \\
\text { unassisted } \\
\text { healing }\end{array}$ & - & - & - & $\begin{array}{l}\text { Only means provided } \\
\text { T } 66.7 \text { mineralised bone; } \\
\text { RGM could not be identified } \\
\text { C } 43.67 \text { mineralised bone }\end{array}$ & - \\
\hline $\begin{array}{l}\text { Vance } \\
\text { et al. } \\
(2004) \dagger\end{array}$ & $\begin{array}{r}\text { Grafting (T1) } \\
\text { vs. GBR (T2) }\end{array}$ & $\begin{array}{c}\mathrm{T} 1-0.3 \pm 0.7 \\
\mathrm{~T} 20.7 \pm 1.2\end{array}$ & $\begin{array}{l}\mathrm{T} 1-0.2 \pm 0.7 \\
\mathrm{~T} 2-0.5 \pm 0.5\end{array}$ & $\begin{array}{l}\mathrm{T} 1-0.5 \pm 0.8 \\
\mathrm{~T} 2-0.5 \pm 0.8\end{array}$ & $\begin{array}{l}\text { T1 } 61 \pm 9 \text { VB; } 3 \pm 3 \text { RGM; } \\
32 \pm 10 \text { trabecular; } 4 \pm 4 \\
\text { amorphous } \\
\text { T2 } 26 \pm 20 \text { VB; } 16 \pm 7 \text { RGM; } \\
54 \pm 15 \text { trabecular; } 5 \pm 6 \\
\text { amorphous }\end{array}$ & $\begin{array}{l}\text { Changes in soft tissue } \\
\text { thickness } \\
\text { T1 Buccal }+0.1 \pm 0.6 ; \\
\text { Lingual }-0.1 \pm 0.7 \\
\text { T2 Buccal }-0.2 \pm 1.5 ; \\
\text { Lingual } 0 \pm 0.7\end{array}$ \\
\hline $\begin{array}{l}\text { Wallace } \\
\text { et al. } \\
\text { (2013) }\end{array}$ & $\begin{array}{l}\text { GBR (T1) vs. } \\
\text { GBR (T2) }\end{array}$ & - & - & - & $\begin{array}{l}\text { Mean (range) } \\
\text { T1 } 41.8(16-66) \text { VB; } 6.6(0-29) \\
\text { RGM;: } 51.6(32-64) \text { marrow/ } \\
\text { CT } \\
\text { T2 } 32.5 \text { (7 to 66) VB; } 16.9 \\
\text { (0-29) RGM; } 50.6(34-65) \\
\text { marrow/CT }\end{array}$ & - \\
\hline $\begin{array}{l}\text { Wood and } \\
\text { Mealey } \\
(2012) \dagger\end{array}$ & $\begin{array}{l}\text { Grafting (T1) } \\
\text { vs. grafting } \\
\text { (T2) }\end{array}$ & $\begin{array}{c}\mathrm{T} 1-0.4 \pm 1.1 \\
\mathrm{~T} 2-0.6 \pm 1.2\end{array}$ & - & $\begin{array}{c}\mathrm{T} 1-2.2 \pm 1.6 \\
\mathrm{~T} 2-2.1 \pm 1.7\end{array}$ & $\begin{array}{l}\text { T1 } 38.4 \pm 14.5 \mathrm{VB} ; 8.9 \pm 12.8 \\
\text { RGM; } 52.7 \pm 8 \mathrm{CT} \\
\text { T2 } 24.6 \pm 13.7 \mathrm{VB} ; 25.4 \pm 17 \\
\text { RGM; } 49.9 \pm 11.1 \mathrm{CT}\end{array}$ & - \\
\hline
\end{tabular}

$\mathrm{VB}$, vital bone; $\mathrm{CT}$, connective tissue; RGM, residual graft material.

Data provided as mean \pm SD unless noted otherwise; patient is unit of analysis unless specified otherwise; dimensional changes provided in millimetres.

All studies included for Question 2; highlighted studies included for Question 1.

*Selected for meta-analysis Question 1.

$\dagger$ Selected for meta-analysis Question 2.

Included only for histological data.

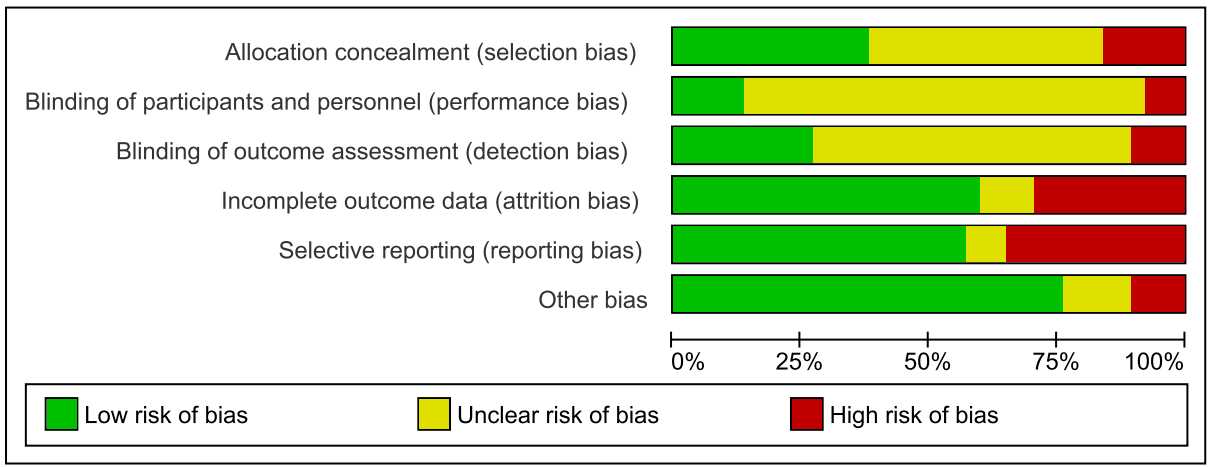

Fig. 2. Meta-analysis results and heterogeneity test for Q1; parallel studies. (a) Parallel studies investigating linear and volumetric changes in vertical alveolar bone height (mid-buccal), (b) split-mouth studies reporting on changes in the mid-buccal vertical alveolar ridge dimensions, (c) parallel studies investigating linear and volumetric changes in vertical alveolar bone height (proximal) and (d) parallel studies investigating linear and volumetric changes in alveolar bone width.

mean difference (SMD) in vertical mid-buccal bone height $(\mathrm{mm})$ between ARP- and non-treated extraction sites was $0.739 \mathrm{~mm}(95 \% \mathrm{CI}$ :

0.332 to 1.147$)$. The difference between the ARP and control groups was found to be statistically significant $(P<0.001)$.
Split-mouth studies: Three studies (Fig. 2) reported data on changes in the mid-buccal vertical alveolar ridge dimensions (Camargo et al. 2000; Festa et al. 2013; Karaca et al. 2015). There was a high level of heterogeneity $\left(I^{2}=76.18 \%, P=0.015\right)$. The standardised mean difference (SMD) in vertical mid-buccal bone height $(\mathrm{mm})$ between ARP- and nontreated extraction sites was $0.975 \mathrm{~mm}$ (95\% CI: 0.017 to 1.933$)$. The difference between the ARP and control groups was found to be statistically significant $(P=0.046)$.

\section{Linear and volumetric changes in vertical} alveolar bone height (proximal)

Parallel studies: Three studies (Fig. 2) reported data on changes in the proximal vertical alveolar ridge dimensions (Iasella et al. 2003; Aimetti et al. 2009; Barone et al. 2013a). There was a low level of heterogeneity 
$\left(I^{2}=24.53 \%, P=0.2658\right)$. The SMD proximal vertical bone height between ARP- and nontreated extraction sites was $0.796 \mathrm{~mm}$ (95\% CI: 0.364 to 1.228 ). The difference between the ARP and control groups was found to be statistically significant $(P<0.001)$.

Split-mouth studies: Only one study (Festa et al. 2013) reported on proximal bone changes in a split-mouth study. The mean change in proximal vertical bone height was $-0.3 \pm 0.8 \mathrm{~mm}$ in the test group and $-0.4 \mathrm{~mm} \pm 1.2$ in the control group. The difference between the measurements was not found to be statistically significant.

\section{Linear and volumetric changes in alveolar} bone width

Parallel studies: Four studies (Fig. 2) reported data on changes in the horizontal alveolar ridge dimensions (Iasella et al. 2003; Aimetti et al. 2009; Barone et al. 2013a; Jung et al. 2013). There was a high level of heterogeneity $\left(I^{2}=91.37 \%, P<0.0001\right)$. The SMD in the horizontal bone width $(\mathrm{mm})$ between ARP and non-treated extraction sites was $1.198 \mathrm{~mm}$ (95\% CI: -0.0374 to 2.433 ). The difference between the ARP and control groups was not found to be statistically significant $(P=0.057)$.

Split-mouth studies: Two studies reported on changes in the horizontal bone measurements. There was a high level of heterogeneity $\left(I^{2}=89.50 \%, P=0.002\right)$. An SMD of -0.161 (95\% CI: -0.866 to 0.544 ) was calculated for Camargo et al. (2000), and 1.478 (95\% CI: 0.652 to 2.304 ) for Festa et al. (2013).

\section{Histological characteristics of new bone formation}

Three studies (Iasella et al. 2003; Fiorellini et al. 2005 and Aimetti et al. 2009) reported on the histological composition of trephined bone core samples after ARP procedures. Aimetti et al. (2009) and Fiorellini et al. (2005) examined the differences in the trabecular bone levels following socket grafting procedures using alloplastic and xenograft/ bioactive materials. Aimetti et al. (2009) found $100 \%$ of living bone in the bone sample following calcium sulphate socket grafting, with $58.8(\mathrm{SD} \pm 3.3) \%$ trabecular bone in the test group and $47.2(\mathrm{SD} \pm 7.7) \%$ in the control group. The difference in the bone content was found to be statistically significant $(P<0.001)$. Greater levels of lamellar bone were found in the test group at coronal and apical sites, with higher levels of woven bone found at the same level in the control group. No inflammation was recorded in either the test or control group samples.
Although Fiorellini et al. (2005) did not report on the exact percentage of new bone formation for the different xenograft materials used, two-thirds of all the collected samples in each test group was found to be trabecular bone,. No evidence of residual collagen matrix was found in the test group, with no difference recorded between the native and induced bone observed. Iasella et al. (2003) found more bone formation in the test group treated with FDBA and a collagen membrane $(65 \pm 10 \%)$ when compared to the unassisted socket healing controls $(54 \pm 12 \%)$. In the test group, $28 \%$ vital and $37 \%$ non-vital FDBA fragments were observed. The residual FDBA particles were often surrounded by woven bone or occasionally encapsulated in fibrous connective tissue. The core samples examined by Fiorellini et al. (2005), Iasella et al. (2003) did not demonstrate the presence of an inflammatory cellular response within the augmented bone.

\section{Changes in keratinised tissue width and thickness}

Three studies reported on the change in the keratinised tissue characteristics following ARP, two studies (Barone et al. 2013a; Festa et al. 2013) following a GBR procedure and one (Iasella et al. 2003) following socket grafting (Table 3). Barone et al. (2013a) reported an increase in the width of the keratinised tissue in both the test and the control groups $(1.14 \pm 0.8 \mathrm{~mm}$ and $0.73 \pm 0.8 \mathrm{~mm})$, with the test group having a greater shift of the gingival tissue towards the occlusal direction after ARP. Iasella et al. (2003) found that a loss in the gingival tissue thickness of -0.1 ( $S D \pm 0.5) \mathrm{mm}$ occurred following GBR using a collagen membrane and allograft material, with a tissue gain of 0.4 $(\mathrm{SD} \pm 0.6) \mathrm{mm}$ in the unassisted control group. The difference between the test and the control groups was found to be statistically significant $(P<0.05)$. Festa et al. (2013) reported on the gingival tissue height following socket grafting using a combination of porcine xenograft and cortical membrane. This study indicated no change to the free gingival margin at the neighbouring teeth following tooth extraction in the test and the control groups.

\section{Post-operative complications and patient-} based outcomes

All nine of the included studies reported on the occurrence of adverse events (Table 4). Five studies (Camargo et al. 2000; Aimetti et al. 2009; Barone et al. 2013a; Festa et al. 2013; Jung et al. 2013) reported no adverse events during the healing phase in the ARP test and control groups. One study did not provide any information on complications (Iasella et al. 2003). Three studies (Fiorellini et al. 2005; Cardaropoli et al. 2014; Karaca et al. 2015) reported a high level of complications in both interventional and control groups. The complications reported in both groups were mainly oedema, oral pain and erythema Fiorellini et al. (2005); Karaca et al. (2015) found that the frequency of these complications was greater in the ARP group. No studies reported on other variables associated with the patient experience in the test or the control group.

\section{Outcome of controlled studies answering focused question 2}

GBR

Linear and volumetric changes in vertical alveolar bone height (mid-buccal)

Meta-analysis of seven studies and eleven subgroups calculated a pooled effect size of $-0.467 \mathrm{~mm}$ (95\% CI: -0.866 to -0.069$)$ reduction in the mid-buccal alveolar ridge height. The degree of variance in the studies was high. Allograft ARP appeared to be associated with a greater range of dimensional change (Iasella et al. 2003; Poulias et al. 2013; Borg \& Mealey 2015).

\section{Proximal vertical bone change}

Meta-analysis of six studies and nine subgroups calculated an effect size of $-0.356 \mathrm{~mm}$ (95\% CI: -0.490 to -0.222 ) reduction in the proximal vertical bone height. The degree of variance in the studies was moderate.

\section{Horizontal changes}

Eight studies with 13 subgroups calculated a pooled effect size of $-1.45 \mathrm{~mm}$ (95\% CI: -1.892 to -1.008 ) reduction in the horizontal bone width. The degree of variance in the studies was high.

\section{Socket grafting}

Vertical mid-buccal bone changes

Nine studies with sixteen subgroups calculated a pooled effect size of $-0.157 \mathrm{~mm}(95 \%$ CI: -0.554 to 0.239 ) reduction in the vertical bone height. The degree of variance in the studies was high. Two studies (Neiva et al. 2008; Coomes et al. 2014) reported positive vertical height changes when the socket graft was covered with a xenograft collagen sponge. 
Table 4. Study outcomes of included papers (II) and Search strategy

\begin{tabular}{|c|c|}
\hline References & Complications \\
\hline \multicolumn{2}{|c|}{ (a) Study outcomes of included papers (II) } \\
\hline Aimetti et al. (2009) & None reported \\
\hline Barone et al. (2013a) & None reported \\
\hline Barone et al. (2013b) & - \\
\hline Barone et al. (2014) & None reported \\
\hline Beck and Mealey (2010) & $\begin{array}{l}\text { T1: post-operative infection ( } 2 \text { sites, ? patients); deficient fill of } \\
\text { socket at } 7 \text { - to } 10 \text {-day follow-up ( } 3 \text { patients) } \\
\text { T2: post-operative infection (1 patient) }\end{array}$ \\
\hline Borg and Mealey (2015) & None reported \\
\hline Calasans-Maia et al. (2014) & None reported \\
\hline Camargo et al. (2000) & None reported \\
\hline Cardaropoli et al. (2014) & Discomfort and swelling were commonly reported in both groups \\
\hline Cook and Mealey (2013) & $\begin{array}{l}\text { T1: apparent post-operative infections at the treatment site that } \\
\text { resolved within } 1 \text { week after switching antibiotic regimens ( } 1 \text { patient) } \\
\text { T2: apparent post-operative infections at the treatment site that } \\
\text { resolved within } 1 \text { week after switching antibiotic regimens } \\
\text { (1 patient), patient reported removing the OP membrane during the } \\
\text { initial } 2 \text { weeks of healing ( } 1 \text { patient) }\end{array}$ \\
\hline Coomes et al. (2014) & $\begin{array}{l}\text { Mild erythema and localised post-operative swelling } 2-3 \text { days after } \\
\text { extraction ( } 12 \% \text { of patients) }\end{array}$ \\
\hline Crespi et al. (2009a) & - \\
\hline Crespi et al. (2011a) & None reported \\
\hline Crespi et al. (2011b) & None reported \\
\hline Eskow \& Mealey (2014) & $\begin{array}{l}\text { T1: } 1 \text { site lost graft particles from the socket during initial healing } \\
\text { which was seen at } 1 \text { week post-operative }\end{array}$ \\
\hline Fernandes et al. (2011) & None reported \\
\hline Festa et al. (2013) & None reported \\
\hline Fiorellini et al. (2005) & $\begin{array}{l}\text { A total of } 250 \text { adverse events were reported for } 78 \text { of } 80 \text { patients. } \\
\text { The most frequent reports were oral oedema }(75 \%) \text {, mouth pain } \\
(68 \%) \text { and oral erythema }(46 \%) \text {. There were a greater number of } \\
\text { cases of oral oedema and erythema in treatment groups compared } \\
\text { to the no treatment group }\end{array}$ \\
\hline Gholami et al. (2012) & None reported \\
\hline Hoang \& Mealey (2012) & $\begin{array}{l}\text { T2: sequestering of some superficial pieces of bone graft material at } \\
\text { the } 1 \text {-week recall ( } 2 \text { patients) }\end{array}$ \\
\hline Huh et al. (2011) & - \\
\hline lasella et al. (2003) & - \\
\hline Jung et al. (2013) & None reported \\
\hline Karaca et al. (2015) & All patients experienced mild to moderate pain at donor site \\
\hline Kim et al. (2014) & No severe adverse events reported \\
\hline Lindhe et al. (2014) & - \\
\hline Mardinger et al. (2012) & - \\
\hline Meloni et al. (2015) & None reported \\
\hline Neiva et al. (2008) & - \\
\hline Mardas (2010) & $\begin{array}{l}\text { Few patients in both groups reported minor post-operative pain or } \\
\text { discomfort, localised oedema and in some cases exfoliated graft } \\
\text { particles were observed. All the patients } \\
\text { presented with membrane exposure at the first post-operative week } \\
\text { that, in most cases, became larger during the second week }\end{array}$ \\
\hline Perelman-Karmon et al. (2012) & None reported \\
\hline Pinho et al. (2006) & $\begin{array}{l}\text { Exposure of membrane in } 5 \text { of the } 10 \text { treated subjects between the } \\
\text { sixth and tenth week of the placements }\end{array}$ \\
\hline Poulias et al. (2013) & None reported \\
\hline Serino et al. (2003) & None reported \\
\hline Vance et al. (2004) & - \\
\hline Wallace et al. (2013) & None reported \\
\hline Wood and Mealey (2012) & $\begin{array}{l}\text { T1: } 2 \text { patients showed signs of potential infection at } 1 \text { week } \\
\text { T2: } 1 \text { patient showed signs of potential infection at } 1 \text { week }\end{array}$ \\
\hline
\end{tabular}

Proximal vertical bone changes

Only two groups from two studies (Vance et al. 2004; Aimetti et al. 2009/ reported on proximal vertical bone changes following socket seal procedures. Meta-analysis was therefore not attempted. A proximal vertical bone height change of $-0.2 \mathrm{~mm}$ was calculated for Aimetti et al. (2009) (95\% CI: -0.451 to 0.051$)$ and $-0.2 \mathrm{~mm}$ for Vance et al. (2004) (95\% CI: -0.596 to 0.196$)$.

\section{Horizontal bone changes}

Eight studies with thirteen subgroups calculated a pooled effect size reduction in the horizontal bone dimension of $-1.613 \mathrm{~mm}$ (95\% CI: -1.989 to -1.238 ). The degree of variance in the studies was moderate.

\section{Socket sealing ARP}

Only two eligible studies (Jung et al. 2013; Meloni et al. 2015) reported on dimensional bone changes following ARP with socket sealing. Their results were found to be divergent. Jung et al. (2013) reported a vertical change of $0 \pm 1.2 \mathrm{~mm}$ and a width reduction of $-1.2 \pm 0.8 \mathrm{~mm}$ following socket seal with a porcine collagen matrix (Mucograft) and a vertical height gain of $1.2 \pm 2.9 \mathrm{~mm}$ and a horizontal reduction of $-1.4 \pm 1 \mathrm{~mm}$ following socket seal with a free gingival graft. Meloni et al. (2015) reported a height reduction of $-1.6 \pm 0.69 \mathrm{~mm}$ and width reduction of $-0.54 \pm 0.25 \mathrm{~mm}$ with a porcine collagen matrix, and height reduction of $-1.47 \pm 0.58 \mathrm{~mm}$ and $-0.67 \pm 0.31 \mathrm{~mm}$ width reduction when using a connective tissue graft. Both studies did not report a statistical difference between the two socket seal interventional groups.

\section{Changes in keratinised tissue width and thickness}

Seven groups from five studies (Iasella et al. 2003; Vance et al. 2004; Barone et al. 2013a, 2014; Festa et al. 2013) reported on keratinised tissue dimensions or gingival tissue thickness following ARP procedures (Table 3). Five groups from four studies (Iasella et al. 2003; Vance et al. 2004; Barone et al. 2013a, 2014) had undergone GBR, with two groups from two studies /Vance et al. 2004; Festa et al. 2013) socket grafting procedures.

Two studies (Barone et al. 2013a, 2014) reported on an increase in the width of keratinised tissue of, respectively, $1.14 \pm 0.8 \mathrm{~mm}$ and $1.18 \pm 0.8 \mathrm{~mm}$ when GBR procedures were carried out. Barone et al. (2014) indicated a reduction in keratinised tissue width $-1.7 \pm 0.6 \mathrm{~mm}$ when a GBR technique was combined with a coronally advanced flap for primary closure. Festa et al. (2013) did not report a change to the keratinised tissue margin when a socket grafting procedure was undertaken.

The thickness of the keratinised tissue margin was reported to be reduced by $-0.1 \pm 0.5 \mathrm{~mm}$ (Iasella et al. 2003) and $-0.2 \pm 1.5 \mathrm{~mm}$ (Vance et al. 2004) when GBR procedures were undertaken. An increase in thickness was reported in a combination grafting procedure (Vance et al. 2004) $0.1 \pm 0.6 \mathrm{~mm}$.

Vance et al. (2004) reported on a reduction $-0.1 \pm 0.7 \mathrm{~mm}$ in the lingual keratinised tissue when a socket grafting procedure was 
Table 4. (continued)

\begin{tabular}{|c|c|c|c|c|c|c|}
\hline & 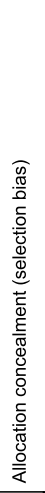 & 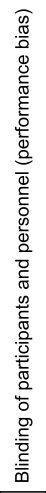 & 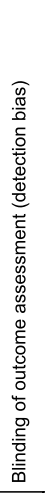 & 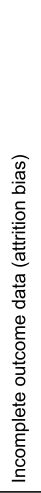 & 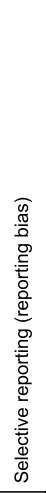 & 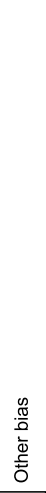 \\
\hline Aimetti 2009 & $?$ & $?$ & $?$ & $\odot$ & $?$ & $?$ \\
\hline Barone 2013a & $?$ & $?$ & $?$ & - & - & + \\
\hline Barone 2013b & + & $?$ & $?$ & - & $\odot$ & + \\
\hline Barone 2014 & + & + & $\odot$ & + & $?$ & + \\
\hline Beck 2010 & - & $O$ & $\odot$ & - & $\odot$ & 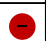 \\
\hline Borg 2015 & + & $?$ & $?$ & + & + & + \\
\hline Calasans-Maia 2014 & + & $?$ & $?$ & + & + & + \\
\hline Camargo 2000 & $?$ & $?$ & $?$ & + & + & + \\
\hline Cardaropoli 2014 & $?$ & $?$ & $\odot$ & + & $?$ & + \\
\hline Cook 2013 & $?$ & $?$ & $?$ & + & $\odot$ & + \\
\hline Coomes 2014 & + & + & $?$ & 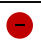 & + & + \\
\hline Crespi 2009 & - & $?$ & $?$ & + & - & + \\
\hline Crespi 2011a & $\theta$ & $?$ & $?$ & + & 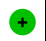 & + \\
\hline Crespi 2011b & $?$ & $?$ & $?$ & + & - & + \\
\hline Eskow 2013 & $?$ & $?$ & $?$ & $\odot$ & 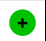 & + \\
\hline Fernandes 2011 & $?$ & $?$ & $?$ & + & + & + \\
\hline Festa 2013 & ? & $?$ & $?$ & 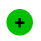 & + & 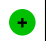 \\
\hline Fiorellini 2005 & + & + & + & $?$ & $\odot$ & $?$ \\
\hline Gholami 2012 & + & $?$ & $\odot$ & + & + & + \\
\hline Hoang 2012 & $?$ & $?$ & $?$ & + & + & $\theta$ \\
\hline Huh 2011 & $?$ & $?$ & $?$ & $?$ & + & + \\
\hline lasella 2003 & $?$ & $?$ & $\odot$ & - & - & + \\
\hline Jung 2013 & + & $?$ & $?$ & $?$ & + & + \\
\hline Karaca 2015 & + & $\theta$ & $\odot$ & + & + & $?$ \\
\hline Kim 2014 & $?$ & $?$ & $?$ & + & + & + \\
\hline Lindhe 2014 & $?$ & $?$ & $?$ & $?$ & $O$ & 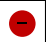 \\
\hline Mardas 2010 & + & + & $\odot$ & + & + & + \\
\hline Mardinger 2012 & $\odot$ & $O$ & $\odot$ & - & + & + \\
\hline Meloni 2015 & + & $?$ & + & + & + & + \\
\hline Neiva 2008 & + & + & + & + & + & + \\
\hline Perelman-Karmon 2012 & $\odot$ & $?$ & $\odot$ & + & - & + \\
\hline Pinho 2006 & + & $?$ & $?$ & + & - & $?$ \\
\hline Poulias 2013 & + & $?$ & $?$ & - & + & + \\
\hline Serino 2003 & ? & $?$ & $?$ & - & - & + \\
\hline Vance 2004 & $?$ & $?$ & $\odot$ & - & - & + \\
\hline Wallace 2013 & - & $?$ & $?$ & + & + & $?$ \\
\hline Wood 2012 & $?$ & $?$ & - & - & $\odot$ & \\
\hline
\end{tabular}

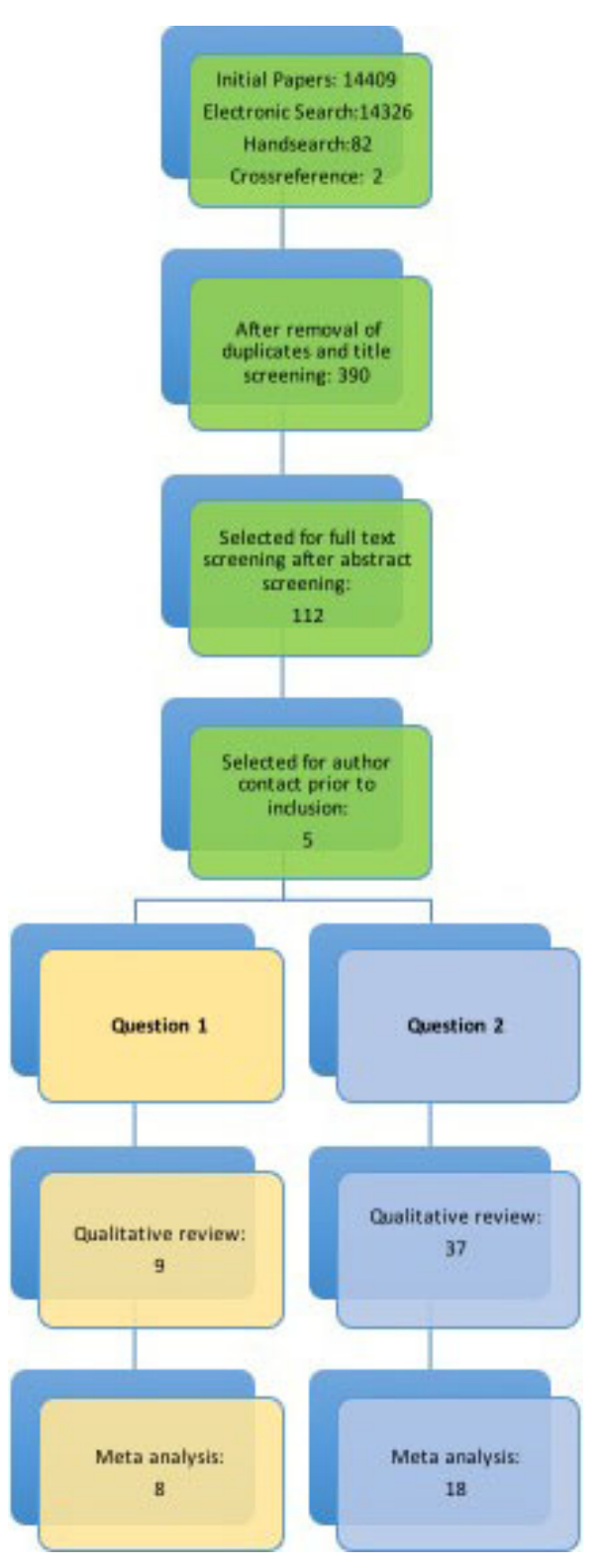

performed but no changes were observed $(0.0 \pm 0.7 \mathrm{~mm})$ when using a GBR procedure.

\section{Histological characteristics of new bone formation}

The histological characteristics of the new tissue, formed within the socket following ARP, were described in 24 studies (Iasella et al. 2003; Serino et al. 2003; Vance et al. 2004; Fiorellini et al. 2005; Neiva et al. 2008; Aimetti et al. 2009; Crespi et al. 2009, 2011a, b; Beck \& Mealey 2010; Mardas et al. 2010; Gholami et al. 2012; Hoang \& Mealey 2012; Mardinger et al. 2012; Perelman-Karmon et al. 2012; Wood \& Mealey 2012; Barone et al. 2013b; Cook \& Mealey 2013; Poulias et al. 2013; Wallace et al. 2013; CalasansMaia et al. 2014; Eskow \& Mealey 2014; Lindhe et al. 2014; Borg \& Mealey 2015).
Only a descriptive analysis was undertaken in this section, as extensive variation was present in the treatment protocols and biomaterials materials used as well as in the histological methods applied to evaluate socket healing. Bone histological samples were reported upon by descriptive analysis, percentage tissue composition (bone/connective tissue/residual particles), mineralised bone content (Aimetti et al. 2009; Gholami et al. 2012; Barone et al. 2013b) and cellular bone composition (cellular/acellular/trabeculla). The included studies reported on the histological characteristics of the trephined core samples over a 10-week to 9month period. The majority of the reports examined histological composition of the core samples at 3 months of healing.

GBR procedures

Seventeen groups from ten studies (Barone et al. 2013b - two groups, Borg \& Mealey 2015 - two groups, Cook \& Mealey 2013 - two groups, Gholami et al. 2012 - two groups, Iasella et al. 2003; Mardas et al. 2010 - two groups, Perelman-Karmon et al. 2012; Poulias et al. 2013 - two groups, Vance et al. 2004; Wallace et al. 2013 - two groups) report on histological composition of bone samples following GBR procedures. The results from these studies showed a high level of variation in the total bone percentage recorded with a range between $47.9 \pm 9.1 \%$ to $24.67 \pm 15.92 \%$ reported. Four studies (Barone et al. 2013b; Borg \& Mealey 2015 - one group, Gholami et al. 2012 - two groups and Vance et al. 2004) reported a total bone composition of $<30 \%$, five (Barone et al. 2013b - one group, Borg \& Mealey 2015 - one group, Cook \& Mealey 2013 - one group, Poulias et al. 2013 - one group and Wallace et al. 2013 - one group) found a $30-40 \%$ bone percentage and four /Cook \& Mealey 2013 - one group, PerelmanKarmon et al. 2012 - one group, Poulias et al. 2013 - one group and Wallace et al. 2013 - one group) reporting over $40 \%$. The use of a combined FDBA and DFDBA (Borg \& Mealey 2015) or collagen/alloplast (Cook \& Mealey 2013) graft produced statistically more bone $(P<0.05)$ when compared with a control using a single allograft or xenograft. The addition of denatured allograft material (Borg \& Mealey 2015) significantly lowered the percentage of residual graft particle $(P=0.035)$. The addition of a bone growth factor also increased the percentage of composition (Wallace et al. 2013). No qualitative differences were recorded between ceramic composite and DBBM (Mardas et al. 2010) or when different xenografts were tested (Barone et al. 2013b). The depth of 
the core sample was found to statistically $(P<0.001)$ influence the bone composition in one study (Perelman-Karmon et al. 2012). Residual and/or encapsulated graft particles were found in five studies (Vance et al. 2004; Mardas et al. 2010; Cook \& Mealey 2013; Wallace et al. 2013; Borg \& Mealey 2015), with the percentage of residual graft particles ranging from 3 to $16.9 \%$.

\section{Socket grafting procedures}

Twenty-four groups from fourteen studies (Aimetti et al. 2009; Beck \& Mealey 2010 two groups, Calasans-Maia et al. 2014 - two groups, Crespi et al. 2009 - two groups, Crespi et al. 2011a - three groups, Crespi et al. 2011b; Eskow \& Mealey 2014 - two groups, Fiorellini et al. 2005 - three groups, Hoang \& Mealey 2012 - two groups, Neiva et al. $2008-$ two groups, Perelman-Karmon et al. 2012 one group, Serino et al. 2003; Vance et al. 2004 and Wood \& Mealey 2012 - two groups) reported on histological composition following various socket grafting procedures. The average trabecular bone composition was recorded by Aimetti et al. (2009) to be $58.8 \%$ with Fiorellini et al. (2005) finding a bone level of $66.6 \%$. Eskow \& Mealey (2014) reported on new bone formation (range 13$16.13 \%)$, Perelman-Karmon et al. (2012) reported on a total bone fraction (range 29.7$40.8 \%$ ), and Serino et al. (2003) measured the average mineralised bone percentage $(66.7 \%)$. The composition of vital bone formation recorded was highly variable, with the percentage recorded ranging from 19.3\% (Beck \& Mealey 2010) to $61 \%$ (Vance et al. 2004). Three studies (Calasans-Maia et al. 2014 one group, Neiva et al. 2008; Vance et al. 2004) reported a vital bone composition of less than $30 \%$, four studies /Calasans-Maia et al. 2014 - one group, Crespi et al. 2011a two groups, Crespi et al. 2011b; Wood \& Mealey 2012) reported a vital bone composition of 30-40\%, and four studies (Beck \& Mealey 2010 - two groups, Crespi et al. 2009 - two groups, Hoang \& Mealey 2012 - two groups and Vance et al. 2004) reported a vital bone composition of more than $40 \%$. No statistical difference was recorded in the vital bone composition when different alloplasts, allografts and xenografts were compared (Beck \& Mealey 2010; Crespi et al. 2011a; Hoang \& Mealey 2012; Calasans-Maia et al. 2014). Eskow \& Mealey (2014) did not observe a statistical difference between cortical or cancellous graft material, and Hoang \& Mealey (2012) failed to observe a difference when using different sized particles of human demineralised bone matrix. A significant difference in the trabecular bone formation was found when human growth hormone or calcium sulphate was added to the graft material (Vance et al. 2004; Fiorellini et al. 2005; Neiva et al. 2008; Crespi et al. 2009). Demineralised freeze-dried allograft generated more vital bone formation when socket grafting using methylcellulose, calcium sulphate and bone allograph was compared against GBR using a bovine xenograft and collagen membrane (Vance et al. 2004). More vital bone was also recorded when socket grafting was undertaken with a demineralised rather than mineralised freezedried bone allograft (Wood \& Mealey 2012). Residual and/or encapsulated graft particles were found in twelve studies (Serino et al. 2003; Vance et al. 2004; Neiva et al. 2008; Crespi et al. 2009, 2011a,b; Beck \& Mealey 2010; Hoang \& Mealey 2012; Wood \& Mealey 2012; Calasans-Maia et al. 2014 and Eskow \& Mealey 2014), with the percentage of residual graft particles ranging from $0 \%$ with a polylactide sponge (Serino et al. 2003) to $36.6 \%$ with a corticocancellous xenogenic graft (Crespi et al. 2011a). No inflammatory response was reported within the histological graft specimens.

\section{Socket seal}

Three groups from two studies (Lindhe et al. 2014 - two groups and Mardinger et al. 2012) reported on bone composition following a socket seal procedure. Lindhe et al. (2014) examined the effect of socket sealing with a collagen membrane or membrane/bovine xenograft combination. Mardinger et al. (2012) evaluated the additional benefit of using the reactive socket tissue as a seal overlying a bovine xenograft. Histological examination by Lindhe et al. (2014) reported $39.9 \pm 8.6 \%$ mineralised bone and $19.5 \pm 6.5 \%$ residual graft in the group combining xenograft with a collagen seal, and $57.4 \pm 12.4 \%$ mineralised bone in the collagen seal alone group. Mardinger et al. (2012) reported $40 \pm 19 \%$ vital bone in the core samples. Three studies (Mardas et al. 2010; Cook \& Mealey 2013; Lindhe et al. 2014) reported fibrous encapsulation of graft particles, with four studies /Crespi et al. 2011b; Hoang \& Mealey 2012; Lindhe et al. 2014; Borg \& Mealey 2015) reporting new bone formation in direct contact with the graft particles with inflammation recorded in the healed overlying gingival tissues.

\section{Histological characteristics of new bone formation}

The depth of the core sample was found to positively influence the composition of new bone formation, with a larger percentage of new bone found in the apical section of the core (Aimetti et al. 2009; Perelman-Karmon et al. 2012). Three studies (Cook \& Mealey 2013; Mardas et al. 2010; Lindhe et al. 2014) reported fibrous encapsulation of graft particles, with four studies (Crespi et al. 2011b; Hoang \& Mealey 2012; Lindhe et al. 2014; Borg \& Mealey 2015) reporting new bone formation in direct contact with the graft particles. No studies reported on signs of inflammation within the histological samples. Although inflammatory cells were a common finding in the core biopsies after ARP, loose graft particles and remnants of the membrane were not usually seen.

Post-operative complications

The presence and absence of complications were reported in twenty-nine studies (Camargo et al. 2000; Serino et al. 2003; Fiorellini et al. 2005; Pinho et al. 2006; Aimetti et al. 2009; Beck \& Mealey 2010; Mardas et al. 2010; Crespi et al. 2011a,b; Fernandes et al. 2011; Gholami et al. 2012; Hoang \& Mealey 2012; Perelman-Karmon et al. 2012; Wood \& Mealey 2012; Barone et al. 2013a, 2014; Cook \& Mealey 2013; Festa et al. 2013; Jung et al. 2013; Poulias et al. 2013; Wallace et al. 2013; Calasans-Maia et al. 2014; Cardaropoli et al. 2014; Coomes et al. 2014; Eskow \& Mealey 2014; Kim et al. 2014; Borg \& Mealey 2015; Karaca et al. 2015; Meloni et al. 2015).

The most common findings were soft tissue inflammation and possible infection (Fiorellini et al. 2005; Beck \& Mealey 2010; Mardas et al. 2010; Wood \& Mealey 2012; Cook \& Mealey 2013; Wallace et al. 2013; Coomes et al. 2014; Karaca et al. 2015). Loose graft particles or deficient socket fill at the ARP site was reported in three socket grafting groups (Beck \& Mealey 2010 - one group, Hoang \& Mealey 2012; Eskow \& Mealey 2014) and one GBR (Mardas et al. 2010) study. Patient discomfort was reported in four studies (Fiorellini et al. 2005; Mardas et al. 2010; Cardaropoli et al. 2014; Karaca et al. 2015). Membrane exposure was recorded in three GBR studies following surgical intervention (Pinho et al. 2006; Mardas et al. 2010; Cook \& Mealey 2013).

\section{Patient-based outcomes}

No studies reported on patient preferences or any other patient-based outcomes following ARP preservations.

\section{Quality assessment \& risk of bias}

The quality assessment of all the included studies for both focused questions is 
presented in Table 4a and b. Only two studies were assessed as having low risk (Neiva et al. 2008; Mardas et al. 2010). Three other studies were assessed as having a low risk of bias in all but one domain (Gholami et al. 2012; Barone et al. 2014; Meloni et al. 2015), and three studies presented with a low risk of bias in four domains (Huh et al. 2011; Calasans-Maia et al. 2014; Borg \& Mealey 2015). All other studies were considered to have an unclear to high risk of bias.

Other sources of bias were the lowest risk category reported when examining the papers, with uncertainty in the blinding of the participants and the outcome assessment, the commonest finding in the papers examined. The highest risk of bias was associated with selective reporting.

\section{Discussion}

\section{Objectives and main findings}

Augmentation procedures have been proposed as a method to limit the adverse functional and volumetric tissue changes experienced during healing after a tooth extraction (Tan et al. 2012; Vignoletti et al. 2012; Mardas et al. 2015). They have been recorded as changing the structural and histological characteristics of the bone and gingival tissue (Block \& Kent 1990; Lindhe et al. 2014; Tan et al. 2012; Horvath et al. 2013; Vignoletti et al. 2014), possibly promoting the establishment of an idealised functional, biologic and aesthetic foundation, before implant-supported or conventional prostheses are provided (Mardas et al. 2015).

The adoption of ARP has been proposed as a method to significantly improve the aesthetic outcome of single-tooth implants, particularly in the anterior maxilla as it has been reported that they may help to retain sufficient bone at dental implant sites to allow fixture placement (Mardas et al. 2015), reduce the risk of subsequent bone loss (Horvath et al. 2013), positively influence the design of the prosthetic tooth (Pagni et al. 2012), improve the emergence profile of the restoration (Belser et al. 2004) and to simplify access for oral hygiene activities. Anticipated soft tissue effects have included an increase in the gingival papilla height and expansion of the fixed keratinised tissue height and width.

Although there is recognition that augmentation protocols can be used to preserve bone and soft tissues, heterogeneity of the published data has led Vignoletti et al. (2012), Horvath et al. (2013), Mardas et al. (2015) to conclude that further research was required to develop a clearer understanding of the variability and characteristics of the clinical outcomes attributed to each grafting procedure, particularly as differences have been recorded in the quality and composition of the supporting bone (Lindhe et al. 2014).

This systematic review has been designed to evaluate the increased available evidence from RCTs, CCT and large prospective case series reporting on ARP procedures and to determine whether additional clarity has been established regarding the advantage and disadvantages of alternative treatment modalities.

This review found significantly less vertical alveolar bone height resorption when ARP was compared to unassisted socket healing. These findings are in agreement with the results published by Horvath et al. (2013), Vignoletti et al. (2011) and Morjaria et al. (2014). Although a mean reduction in alveolar bone width resorption of $1.20 \mathrm{~mm}$ was recorded, this observation was not found to be statistically significant when compared to unassisted socket healing. This conclusion is at odds with that reported by Vignoletti et al. (2011), but the difference may be accounted for by the heterogeneity of the included data, the methodological structure of the review and the limited number of included trials reporting on this finding. When this study was compared with the systematic review performed by Vignoletti et al. (2012), it was found that seven of the twelve studies identified by Vignoletti et al. (2012), did not perform statistical analysis and that three of the remaining five studies were excluded from this systematic review due to insufficient patient numbers, duplicate reporting and incompatible study design.

Histologically, an increase in bone content was found in the ARP group in comparison with the control group. This was also reported in the systematic review by De Risi et al. (2013) and in certain groups in the systematic review by Horvath et al. (2013). ARP studies, however, reported a higher level of complications and an increased frequency of oedema, facial pain and erythema (Fiorellini et al. 2005; Karaca et al. 2015).

No studies reported on other variables associated with the patient experience.

Although various surgical techniques and materials have been used for ARP, no material or type of ARP intervention can be claimed to yield superior results to another (De Risi et al. 2013; Horvath et al. 2013; Mardas et al. 2015). Previous systematic reviews concluded that the use of barriers for GBR appeared to be more effective in limiting post-extraction dimensional changes of the alveolar ridge /Vignoletti et al. 2012; Horvath et al. 2013; Avila-Ortiz et al. 2014). Although direct statistical comparison was not possible, a greater vertical bone dimensional change was recorded following GBR when compared with the dimensional findings for socket grafting. The amount of horizontal bone dimensional change was noted to be greater with socket grafting than that reported for GBR procedures.

Keratinised tissue measurements were not commonly reported following ARP procedures. This is surprising as the conservancy of the fixed keratinised tissues might affect long-term peri-implant health and decrease the risk for biologic complications (Tan et al. 2012) if an implant-supported restoration is considered. GBR techniques appeared to result in an increase in the keratinised tissue width when no attempt at primary closure was undertaken. No change in the soft tissue width was reported when socket grafting was used. The thickness of the gingival tissues was slightly reduced with GBR procedures (Iasella et al. 2003; Vance et al. 2004), with a small gain noted when using a combination of collagen/particulate socket graft (Vance et al. 2004).

The use of GBR or socket grafting techniques in this systematic review seemed to produce a similar range of bone composition (vital and trabecular bone) in histological samples. The effect of using different GBR, socket grafting materials and particle size on new bone formation was inconclusive, as no statistical advantage was reported in the reviewed RCTs. Demineralised freeze-dried bone was reported as having a statistical influence on the creation of the new vital bone fraction in socket grafting techniques. More vital bone was reported in the apical area of core samples, when compared with coronal sections.

The depth of the core sample was found to influence the bone composition (Aimetti et al. 2009; Perelman-Karmon et al. 2012). Although the presence of residual graft particle has been recorded as interfering or disrupting the process of bone healing, only three of the twentyfour studies (Mardas et al. 2010; Cook \& Mealey 2013; Lindhe et al. 2014) reported on fibrous encapsulation of the graft particles, with no studies reporting on inflammation within the core samples.

The incidence of complications reported within the ARP studies was low. Loose graft particle or deficient socket fill was the commonest adverse event in socket graft 
procedures (Beck \& Mealey 2010; Hoang \& Mealey 2012; Eskow \& Mealey 2014), with exposure of the membrane reported in three of ten included GBR studies. An exposure of the graft particle was associated with the presence of fibrous encapsulation within the histological specimen (Cook \& Mealey 2013).

\section{Strength and weakness of the systematic review}

As in the previous systematic review by our group (Mardas et al. 2015), two focus questions were formulated to try to ensure that all available relevant information on ARP was included in the study. The first focused question limited inclusion to RCTs, CCTs and prospective cohort studies with a control group of unassisted socket healing, in order to identify comparative site dimensional and qualitative tissue effects following ARP procedures. This was based on the fact that the clinical merit of applying ARP is based on the assumption that they will have an additional positive effect on tissue conservancy and bone characteristics over unassisted healing and will validate use of the procedure. For the second focused question, controlled studies without a control group and large prospective case series were also included, to ensure that as much of the available published data was used to estimate pooled tissue changes according to three types of interventions for ARP.

Although a comprehensive search strategy including five databases, extensive handsearch and cross-reference search and no language restriction were applied, it is possible that some grey literature may not have been included as only published studies were selected. In order to obtain as much data as possible from published studies, the authors of five studies selected for full-text screening were contacted via email to request further information relating to the dimensional and histological changes following ARP. Some authors failed to respond within the requested period of time; therefore, it is possible that further information exists which could be used to complement the data set used in this review.

The total number of subjects and selected studies for focused questions 1 and 2 could be considered sufficient for the assessment of effect size differences between ARP and unassisted socket healing and to calculate mean bone and soft tissue dimensional changes following GBR and socket grafting ARP procedures. Limited data was available, however, to evaluate the influence of socket seal techniques on site dimensional changes, histological characteristics and patient outcome factors and as a result the findings in this section of the analysis should be interpreted with caution. Finally, the sample sizes of all the selected clinical trials were relatively small, with many not including a sample size calculation. This may have reduced the power of the studies.

\section{Confounding factors}

Socket wall

As the majority of studies in this systematic review had at least three walls of the socket intact, with more than $50 \%$ of the fourth wall remaining intact, the impact of socket wall integrity on the ARP outcome is relatively unknown. The tooth extraction sites were recorded as being heterogeneous, minimising the effect of the position of the extracted teeth on the outcomes.

\section{Measurements}

The method used to measure the alveolar bone dimensions varied in several studies. Twenty-two of the 27 included studies used direct measurements from static casts, in preference to CBCT radiographic images. As static cast measurements can be influenced by the impression technique and soft tissue changes, difference in the effect of the intervention may have occurred. The possible variation that this may have caused in the recorded measurements was not considered in this review.

\section{Patient-based outcomes}

As a significant number of publications did not report on this finding, then there may be a higher risk of under-reporting.

\section{Antimicrobial use}

Antibiotics were commonly prescribed as an adjunct to ARP, with extensive variation in prescription pattern, dose and length of use. Antibiotic prophylaxis in alveolar and implant surgical procedure has been shown to have a small statistic effect on healing and outcome Esposito et al. (2008). The impact of this variable was not considered as a component of this review.

\section{Conclusion}

Within the limitations of this study, the following conclusions can be drawn:

- ARP results in a significant reduction in the vertical bone dimensional change following tooth extraction when compared to unassisted socket healing.
- A reduction in horizontal alveolar bone dimensional change was found when ARP was compared to unassisted socket healing, but the difference between techniques was not found to be statistically significant.

- No evidence was identified to clearly indicate the superior impact of a type of ARP intervention $(\mathrm{GBR}$, socket filler and socket seal) on bone dimensional preservation or keratinised tissue dimensions. Currently, it is not known whether a biomaterial or a treatment protocol is superior to others.

- There is insufficient evidence to demonstrate a difference in the amount of vital bone formation following GBR or socket grafting techniques.

- Inflammation was common following ARP.

- The majority of the studies evaluating ARP procedures presented with high or unclear risk of bias. Clinical recommendations derived from this study should be interpreted with caution.

\section{Recommendations for further research}

- There is still a need for high-quality RCTs on adequately powered sample sizes to evaluate differences in outcomes between different ARP procedures and unassisted socket healing. Socket seal procedures should be further investigated in comparison with other ARP interventions.

- The role of possible confounding factors such as smoking, reason for extraction, tooth type and location, integrity of buccal bone plate, flap reflection and closure and antibiotic usage should be further investigated.

- Patient-based outcomes and cost-benefit indicators should be included in future trials.

In all future trials, special emphasis should be given to the following issues:

1. Decrease in heterogeneity and control of reported sources of bias.

2. Radiographic assessment of marginal bone levels should be performed on standardised radiographs taken at specific period of times.

3. Soft tissue dimensional measurement should be standardised by using modern technologies like 3D computer-aided analysis. 


\section{References}

Adriaens, P.A. \& Van der Stede, N. (1998) Alveolar bone protection of extraction sites with a resorbable bilayer GBR membrane. Journal of Dental Research 77: 777-790.

Aimetti, M., Romano, F., Griga, F. \& Godio, L. (2009) Clinical and histologic healing of human extraction sockets filled with calcium sulphate. The International Journal of Oral «) Maxillofacial Implants 24: 902-909.

Amler, M.H. (1969) The time sequence of tissue regeneration in human extraction wounds. Oral Surgery Oral Medicine Oral Pathology 27: 309318.

Araujo, M.G., Da Silva, J.C., De Mendonca, A.F. \& Lindhe, J. (2015) Ridge alterations following grafting of fresh extraction sockets in man. A randomized clinical trial. Clinical Oral Implants Research 26: 407-412.

Artzi, Z., Tal, H. \& Dayan, D. (2000) Porous bovine bone mineral in healing of human extraction sockets. part 1: histomorphometric evaluations at 9 months. Journal of Periodontology 71: 10151023.

Avila-Ortiz, G., Rodriguez, J.C., Rudek, I., Benavides, E., Rios, H. \& Wang, H.L. (2014) Effectiveness of three different alveolar ridge preservation techniques: a pilot randomized controlled trial. The International Journal of Periodontics and Restorative Dentistry 34: 509-521.

Barone, A., Ricci, M., Tonelli, P., Santini, S. \& Covani, U. (2013a) Tissue changes of extraction sockets in humans: a comparison of spontaneous healing vs. ridge preservation with secondary soft tissue healing. Clinical Oral Implants Research 24: 1231-1237.

Barone, A., Todisco, M., Ludovichetti, M., Gualini, F., Aggstaller, H. \& Torres- Lagares, D. (2013b) A prospective, randomized, controlled, multi-centre evaluation of extraction socket preservation comparing two bovine xenografts: clinical and histologic outcomes. The International Journal of Periodontics and Restorative Dentistry 33: 795 802.

Barone, A., Toti, P., Piattelli, A., Iezzi, G., Derchi, G. \& Covani, U. (2014) Extraction socket healing in humans after ridge preservation techniques: comparison between flapless and flapped procedures in a randomized clinical trial. Journal of Periodontology 85: 14-23.

Bartee, B.K. (2001) Extraction site reconstruction for alveolar ridge preservation. Part 2: membraneassisted surgical technique. Journal of Oral Implantology 27: 194-197.

Beck, T.M. \& Mealey, B.L. (2010) Histologic analysis of healing after tooth extraction with ridge preservation using mineralized human bone allograft. Journal of Periodontology 81: 1765 1772.

Becker, W., Urist, M., Becker, B., Jackson, W., Pony, D., Bartold, M., Vincenzzi, G., De Georges, D. \& Niederwanger, M. (1996) Clinical and histological observations of sites implanted with intraoral autologous bone grafts or allografts. 15 human case reports. Journal of Periodontology 67: 10251033.
Belser, U., Buser, D. \& Higginbottom, F. (2004) Consensus statements and recommended clinical procedures regarding aesthetics in implant dentistry. International Journal of Oral «) Maxillofacial Implants 19(Suppl): 73-74.

Block, M.S. \& Kent, J.N. (1990) Factors associated with soft and hard-tissue compromise of endosseous implants. Journal of Oral Maxillofacial Surgery 48: 1153-1160.

Borenstein, M., Hedges, L., Higgins, J. \& Rothstein, H. (2009) Introduction to Meta- Analysis. London: Wiley.

Borg, T.D. \& Mealey, B.L. (2015) Histologic healing following tooth extraction with ridge preservation using mineralized versus combined mineralizeddemineralized freeze-dried bone allograft: a randomized controlled clinical trial. Journal of Periodontology 86: 348-355.

Calasans-Maia, M., Resende, R., Fernandes, G., Calasans-Maia, J., Alves, A. \& Granjeiro, J. (2014) A randomised controlled clinical trial to evaluate a new xenograft for alveolar socket preservation. Clinical Oral Implant Research 25: 1125-1130.

Camargo, P., Lekovic, V., Weinlaender, M., Klokkevold, P., Kenny, E., Dimitrijevic, B., Nedic, M., Jancovic, S. \& Orsini, M. (2000) Influences of bioactive glass in alveolar process dimensions after exodontia. Oral Surgery Oral Medicine Oral Pathology Oral Radiology Endodontics 90: 581586.

Cardaropoli, D., Tamagnone, L., Roffredo, A. \& Gaveglio, L. (2014) Relationship between the buccal bone plate thickness and the healing of post extraction sockets with/without ridge preservation. The International Journal of Periodontics and Restorative Dentistry 34: 211-217.

Cook, D.C. \& Mealey, B.L. (2013) Histologic comparison of healing following tooth extraction with ridge preservation using two different xenograft protocols. Journal of Periodontology 84: 585-594.

Coomes, A.M., Mealey, B.L., Huynh-Ba, G., Barboza-Arguello, C., Moore, W.S. \& Cochran, D.L. (2014) Buccal bone formation after flapless extraction: a randomized, controlled clinical trial comparing recombinant human bone morphogenetic protein 2/absorbable collagen carrier and collagen sponge alone. Journal of Periodontology 85: 525535 .

Crespi, R., Cappare, P. \& Gherlone, E. (2009) Magnesium-enriched hydroxyapatite compared to calcium sulfate in the healing of human extraction sockets: radiographic and histomorphometric evaluation at 3 months. Journal of Periodontology 80: 210-218.

Crespi, R., Cappare, P. \& Gherlone, E. (2011a) Comparison of magnesium-enriched hydroxyapatite and porcine bone in human extraction socket healing: a histologic and histomorphometric evaluation. The International Journal of Oral «) Maxillofacial Implants 26: 1057-1062.

Crespi, R., Cappare, P., Romanos, G.E., Mariani, E., Benasciutti, E. \& Gherlone, E. (2011b) Corticocancellous porcine bone in the healing of human extraction sockets: combining Histomorphometry with osteoblast gene expression profiles in vivo.
The International Journal of Oral \&) Maxillofacial Implants 26: 866-872.

Darby, I., Chen, S.T. \& Buser, D. (2009) Ridge preservation techniques for implant therapy. International Journal of Oral Maxillofacacial Implants 24(Suppl): 260-271.

De Risi, V., Clementini, M., Vittorini, G., Mannocci, A. \& De Sanctis, M. (2013) Alveolar ridge preservation techniques: a systematic review and meta-analysis of histological and histomorphometrical data. Clinical Oral Implants Research. 119.

Eskow, A.J. \& Mealey, B.L. (2014) Evaluation of healing following tooth extraction with ridge preservation using cortical versus cancellous freeze-dried bone allograft. Journal of Periodontology 85: 514-524.

Esposito, M., Cannizzaro, G. \& Bozzoli, P. (2008) Efficacy of prophylactic antibiotics for dental implants: a multi centre placebo-controlled randomised clinical trial. European Journal Oral Implantology 1: 23-31.

Farmer, M. \& Darby, I. (2014) Ridge dimensional changes following single-tooth extraction in the aesthetic zone. Clinical Oral Implants Research 25: 272-277.

Fernandes, P.G., Novaes, A.B., de Queiroz, A.C., de Souza, S.L.S., Taba, M., Palioto, D.B. \& Grisi, M. F. (2011) Ridge preservation with acellular dermal matrix and anorganic bone matrix cell-binding peptide P-15 after tooth extraction in humans. Journal of Periodontology 82: 72-79.

Festa, V.M., Addabbo, F., Laino, L., Femiano, F. \& Rullo, R. (2013) Porcine-derived xenograft combined with a soft cortical membrane versus extraction alone for implant site development: a clinical study in humans. Clinical Implant Dentistry and Related Research 15: 707-713.

Feuille, F., Knapp, C., Brunsvold, M. \& Mellonig, J. (2003a) Clinical and histologic evaluation of bone-replacement grafts in the treatment of localized alveolar ridge defects. Part 1: mineralized freeze-dried bone allograft. International Journal of Periodontics «) Restorative Dentistry 23: 2835.

Feuille, F., Knapp, C.I., Brunsvold, M.A. \& Mellonig, J.T. (2003b) Clinical and histologic evaluation of bone- replacement grafts in the treatment of localized alveolar ridge defects. Part 1: mineralized freeze-dried bone allograft. International Journal Periodontics Restorative Dentistry 23: 29-35.

Fiorellini, J.P., Howell, T.H., Cochran, D., Malmquist, J., Lilly, L.C., Spagnoli, D., Toljanic, J., Jones, A. \& Nevins, M. (2005) Randomized study evaluating recombinant human bone morphogenetic protein-2 for extraction socket augmentation. Journal of Periodontology 76: 605613.

Froum, S., Cho, S.-C., Rosenberg, E., Rohrer, M. \& Tarnow, D. (2002) Histological comparison of healing extraction sockets implanted with bioactive glass or demineralized freeze-dried bone allograft: a pilot study. Journal of Periodontology 73: 94-102. 
Garg, A.K. (2001) Preservation, augmentation, and reconstruction of the alveolar ridge. Dental Implantology Update 12: 81-85.

Gholami, G.A., Najafi, B., Mashhadiabbas, F., Goetz, W. \& Najafi, S. (2012) Clinical, histologic and histomorphometric evaluation of socket preservation using a synthetic nanocrystalline hydroxyapatite in comparison with a bovine xenograft: a randomized clinical trial. Clinical Oral Implants Research 23: 1198-1204.

Gross, J. (1995) Ridge preservation using HTR synthetic bone following tooth extraction. General Dentistry 43: 364-367.

Higgins, J.P.T. \& Green, S. (2011) Cochrane Handbook for Systematic Reviews of Interventions. Version 5.1.0 [updated March 2011]. Chichester: The Cochrane Collaboration, 2011. Available from www.cochrane-handbook.org (accessed 1 May 2015)

Higgins, J.P., Thompson, S.G., Deeks, J.J. \& Altman, D.G. (2003) Measuring inconsistency in meta-analyses. British Medical Journal 327: 557560.

Hoang, T.N. \& Mealey, B.L. (2012) Histologic comparison of healing after ridge preservation using human demineralized bone matrix putty with one versus two different-sized bone particles. Journal of Periodontology 83: 174-181.

Horvath, A., Mardas, N., Mezzomo, L.A., Needleman, I.G. \& Donos, N. (2013) Alveolar ridge preservation. A systematic review. Clinical Oral Investigations 17: 341-363.

Hsun-Liang, C., Guo-Hao, L., Jia-Hui, F. \& HomLay, W. (2013) Alteration in bone quality after socket preservation with grafting materials: a systematic review. The International Journal of Oral and Maxillo-Facial Implants 28: 710720.

Huh, J., Lee, H., Jang, J., Kim, M., Yun, P., Kim, S., Choi, K., Cho, K. \& Shin, S. (2011) Randomised clinical trial on the efficacy of escherichia coliderived rhbmp-2 with B-TCP/HA in extraction socket. The Journal of Advanced Prosthodontics 3: 161-165.

Iasella, J.M., Greenwell, H., Miller, R.L., Hill, M., Drisko, C., Bohra, A.A. \& Scheetz, J.P. (2003) Ridge preservation with freeze-dried bone allograft and a collagen membrane compared to extraction alone for implant site development: a clinical and histologic study in humans. Journal of Periodontology 74: 990-999.

Johnson, K. (1969) A study of the dimensional changes occurring in the maxilla following tooth extraction. Australian Dental Journal 14: 241-244.

Jung, R.E., Fenner, N., Hammerle, C.H.F. \& Zitzmann, N.U. (2013) Long-term outcome of implants placed with guided bone regeneration (GBR) using resorbable and non-resorbable membranes after 12-14 years. Clinical Oral Implants Research 24: 1065-1073.

Jung, R.E., Siegenthaler, D.W. \& Hammerle, C.H. (2004) Post extraction tissue management: a soft tissue punch technique. International Journal of Periodontics and Restorative Dentistry 24: 545553.

Karaca, Ç., Er, N., Gülşahi, A. \& Köseoğlu, O. (2015) Alveolar ridge preservation with a free gingival graft in the anterior maxilla: volumetric evaluation in a randomized clinical trial. International Journal Oral Maxillofacial Surgery 44: 774-780.

Kim, Y., Lee, J., Kim, J., Park, J., Shin, S. \& Choo, K. (2014) Ridge preservation using demineralized bone matrix gel with recombinant human bone morphogenic protein-2 after tooth extraction: a randomised controlled clinical trial. Journal of Oral Maxillofacial Surgery 77: 1281-1291.

Lekovic, V., Camargo, P.M., Klokkevold, P.R., Weinlaender, M., Kenney, E.B., Dimitrijevic, B. \& Nedic, M. (1998) Preservation of alveolar bone in extraction sockets using bioabsorbable membranes. Journal of Periodontology 69: 1044-1049.

Lindhe, J., Cecchinato, D., Donati, M., Tomasi, C. \& Liljenberg, B. (2014) Ridge preservation with the use of deproteinized bovine bone mineral. Clinical Oral Implants Research 25: 786-790.

Mardas, N., Chadha, V. \& Donos, N. (2010) Alveolar ridge preservation with guided bone regeneration and a synthetic bone substitute or a bovinederived xenograft: a randomized, controlled clinical trial. Clinical Oral Implants Research 21: 688-698.

Mardas, N., Trullenque-Eriksson, A., MacBeth, N., Petrie, A. \& Donos, N. (2015) Does ridge preservation following tooth extraction improve implant treatment outcomes: a systematic review. Clinical Oral Implant Research. 1-12.

Mardinger, O., Vered, M., Chaushu, G. \& Nissan, J. (2012) Histomorphometrical analysis following augmentation of infected extraction sites exhibiting severe bone loss and primarily closed by intrasocket reactive soft tissue. Clinical Implant Dentistry and Related Research 14: 359-365.

Meloni, S.M., Tallarico, M., Lolli, F.M., Deledda, A., Pisano, M. \& Jovanovic, S.A. (2015) Post extraction socket preservation using epithelial connective tissue graft vs porcine collagen matrix. 1-year results of a randomised controlled trial. European Journal of Oral Implantology 8: 39-48.

Morjaria, K.R., Wilson, R. \& Palmer, R.M. (2014) Bone healing after tooth extraction with or without an intervention: a systematic review of randomized controlled trials. Clinical Implant Dentistry and Related Research 16: 1-20.

Neiva, R.F., Tsao, Y.-P., Eber, R., Shotwell, J., Billy, E. \& Wang, H.-L. (2008) Effects of a putty-form hydroxyapatite matrix combined with the synthetic cell- binding peptide P-15 on alveolar ridge preservation. Journal of Periodontology 79: 291299.

Pagni, G., Pellegrini, G., Giannobile, W. \& Rasperini, G. (2012) Post extraction alveolar ridge preservation: biological basis and treatment. International Journal of Dentistry 2012: 151030: 0-13.

Perelman-Karmon, M., Kozlovsky, A., Liloy, R. \& Artzi, Z. (2012) Socket site preservation using bovine bone mineral with and without a bioresorbable collagen membrane. The International Journal of Periodontics and Restorative Dentistry 32: 459-465.

Pinho, M.N., Roriz, V.L.M., Novaes, A.B., Jr, Taba, M., Jr, Grisi, M.F.M., De Souza, S.L.S., et al.
(2006) Titanium membranes in prevention of alveolar collapse after tooth extraction. Implant Dentistry 15: 53-61.

Poulias, E., Greenwell, H., Hill, M., Morton, D., Vidal, R., Shumway, B. \& Peterson, T.L. (2013) Ridge preservation comparing socket allograft alone to socket allograft plus facial overlay xenograft: a clinical and histologic study in humans. Journal of Periodontology 84: 1567-1575.

Prato, G.P., Cairo, F., Tinti, C., Cortellini, P., Muzzi, L. \& Mancini, E.A. (2004) Prevention of alveolar ridge deformities and reconstruction of lost anatomy: a review of surgical approaches. International Journal Periodontics Restorative Dentistry 24: 434-445.

Schropp, L., Isidor, F., Kostopoulos, L. \& Wenzel, A. (2005) Interproximal papilla levels following early versus delayed placement of a single-tooth implants: a controlled clinical trial. International Journal Oral Maxillofacial Implants 20: 753-761.

Seibert, J.S. (1983) Reconstruction of deformed, partially edentulous ridges, using full thickness onlay grafts. Part I. technique and wound healing. Compendium continuing education. Dentistry 4: 437-453.

Serino, G., Biancu, S., Iezzi, G. \& Piattelli, A. (2003) Ridge preservation following tooth extraction using a polylactide and polyglycolide sponge as space filler: a clinical and histological study in humans. Clinical Oral Implants Research 14: 651-658.

Studer, S.P., Lehner, C., Bucher, A. \& Scharer, P. (2000) Soft tissue correction of a single-tooth pontic space: a comparative quantitative volume assessment. Journal Prosthetic Dentistry 83: 402411.

Tan, W.L., Wong, T.L.T., Wong, M.C.M. \& Lang, N.P. (2012) A systematic review of post-extractional alveolar hard and soft tissue dimensional changes in humans. Clinical Oral Implants Research 23: 1-21.

Tarnow, D., Eskow, R. \& Zamzok, J. (1996) Aesthetic and implant dentistry. Periodontology 2000 11: 85-94.

Thoma, D.S., Benic, G.I., Zwahlen, M., Hammerle, C.H. \& Jung, R.E. (2009) A systematic review assessing soft tissue augmentation techniques. Clinical Oral Implants Research 4: 146165.

Vance, G.S., Greenwell, H., Miller, R.L., Hill, M., Johnston, H. \& Scheetz, J.P. (2004) Comparison of an allograft in an experimental putty carrier and a bovine-derived xenograft used in ridge preservation: a clinical and histologic study in humans. The International Journal of Oral \&) Maxillofacial Implants 19: 491-497.

Vignoletti, F., Matesanz, P., Rodrigo, D., Figuero, E., Martin, C. \& Sanz, M. (2012) Surgical protocols for ridge preservation after tooth extraction. A systematic review. Clinical Oral Implants Research 23: 22-38.

Vignoletti, F., Nunez, J. \& Sanz, M. (2014) Soft tissue wound healing at teeth, dental implants and the edentulous ridge when using barrier membranes, growth and differentiation factors and soft tissue substitutes. Journal of Clinical Periodontology 41: S23-S35. 
Wallace, S., Snyder, M. \& Prasad, H. (2013) Post extraction ridge preservation and augmentation with mineralized allograft with or without recombinant human platelet-derived growth factor $\mathrm{Bb}$ (Rhpdgf-Bb): a consecutive case series. The International Journal of Periodontics and Restorative Dentistry 33: 599-609.
Wang, H., Kiyonobu, K. \& Neiva, R. (2004) Socket augmentation: rationale and technique. Implant Dentistry 13: 286-296.

Wang, R.E. \& Lang, N.P. (2012) Ridge preservation after tooth extraction. Clinical Oral Implants Research 23: 147-156.
Wood, R.A. \& Mealey, B.L. (2012) Histologic comparison of healing after tooth extraction with ridge preservation using mineralized versus demineralized freeze-dried bone allograft. Journal of Periodontology 83: 329-336. 